\title{
II. JAHIBESBERICHTE.
}

46. Die griechischen historiker der späteren zeit.

I. Dionysius von Halikarnass.

Erster abschnitt.

1. Dionysii Halicarnassensis opera omnia graece et latine cum annotationibus H. Stephani, Frid. Sylburgii, Franc. Porti, Is. Casauboni, Fulvii Ursini, Henr. Valesii, Jo. Hudsoni et Jo. Jac. Reiske. VI voll. 8. Lips. in libraria Weidmannia 1774-77.

2. Dionysii Halicarnassensis opera omnia quibus etiam accedunt fragmenta ab A. Majo nuper reperta. Ed. stereotypa. 6 voll. 16. Lips. Tauchnitz. 1823 (29).

3. David Christianus Grimm Lectionum Dionysianarum fasc. I-IV. Annaberg. 4. 1783-85.

4. Dionysii Halicarnassensis archaeologiae romanae quae ritus romanos explicat synopsis. Adornavit David Chr. Grimm. Accedunt auctaria quaedam. 8. Lips. sumtu Weidmanni hered. et Reichii. 1786.

5. J.S. Horstig, explicatio Dionysii Halicarnassensis archaeologiae romanae lib. X c. I-VII. 8. Lips. 1790.

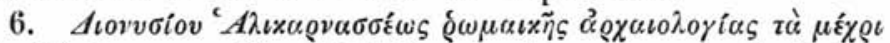
$\tau o v ้ \delta \varepsilon \dot{\varepsilon} \lambda \lambda \varepsilon i \pi o v \tau \alpha$. Dionysii Halicarnassei romanarum antiquitatum pars hactenus desiderata, nunc denique ope codd. Ambrosianorum ab Angelo Majo quantum licuit restituta. 8. Mediol. 1816 (Francofurti ad M. 1817).

7. C. L. Struve, über die von Majus in Mailand aufgefundenen und herausgegebenen bruchstücke des Dionysius von Halicarnass. 8. Königsberg. 1820 . $-57 \mathrm{~s}$.

8. C. L. Struve, über die zweite von Majus besorgte ausgabe der fragmenta des Dionysius von Halicarnass, in Jahn jahrb. f. phil. u. paed. VII (1828) p. $363 \mathrm{ff}$.

9. Ph. F. Schulin de Dionysio Halicarnassensi historico, praecipuo iuris romani fonte. 4. Heidelb. 1820. - $97 \mathrm{~s}$.

Philologus. XXXVI. bd. 1. 
10. C. G. Weissmann de Dionysii Halicarnassensis vita et ingenio. 4. Rinteln. 1837.

11. Dionysii Halicarnassensis prooemium antiquitatum romanarum e codd. mss., de quorum indole et usu disputatur, emendatum a $\mathrm{Fr}$. Ritschelio. Accedit tabula lithographica. 4. Vratisl. 1838. - 28 s.

12. Dionysii Halicarnassensis antiquitatum romanarum capp. undetriginta quae ad instituta et leges Romuli regis pertinent, e codd. mss. emendavit et annotatione critica instruxit J. $A$. Ambrosch. 4. Vratislav. Hirt. 1840. - 38 s.

13. Eclogae ambrosianae ad Dionysii Halicarnassensis antiquitatum romanarum librum $\mathbf{X}$. pertinentes e codd. mss. edidit et annotatione instruxit J.A. Ambrosch. 4. Vratisl. 1841. - $18 \mathrm{~s}$.

14. Ant. Guil. Ferd. Busse de Dionysii Halicarnassensis vita et ingenio dissertatio. 4. Berol. 1841. - $62 \mathrm{~s}$.

15. J. A. Ambrosch, Quaestionum ad Dionysii Halicarnassei antiquitates romanas pertinentium particula prima. 4. Vratisl. 1842. $-24 \mathrm{~s}$.

16. J. A. Ambrosch, Quaestionum ad Dionysii Halicarnassei antiquitates romanas pertinentium particula altera. 4. Vratisl. 1843. $-8 \mathrm{~s}$.

17. J. A. Ambrosch, Ex Dionysii Halicarnassei antiquitatibus romanis capita, quae ad res Romuli pertinent e codd. mss. emendata. 4. Vratisl. 1844. $-28 \mathrm{~s}$.

18. J. A. Ambrosch, Ex Dionysii Halicarnassei antiquitatibus romanis capita quae sacerdotia Numae continent e codd. mss. emendata. 4. Vratisl. 1845. $-17 \mathrm{~s}$.

19. J. A. Ambrosch, Ex Dionysii Halicarnassei antiquitatibus historiae gentis romanae particula prior e codd, mss. emendata. 4. Vratisl. 1846. - 33 s.

20. J. Kuschel, de fontibus et auctoritate Dionysii Halicarnassei. 8. part. I. Vratisl. 1846.

21. Dionysii Halicarnassensis antiquitatum romanarum libri I capita XXX priora ex optimis codd. emendata a Frid. Ritschelio. 4. Bonnae. 1846. - $41 \mathrm{~s}$.

22. Fr. Ritschelii de codice Urbinate Dionysii Halicarnassensis disputatio. 4. Bonnae. 1847. $-25 \mathrm{~s}$.

23. C. G. Cobet oratio de arte interpretandi grammatices et critices fundamentis innixa primario philologi officio, 8. Lugduni Bat. 1847.

24. Fragmenta historicorum graecorum. Collegit, disposuit, notis et prolegomenis illustravit, indicibus instruxit Carolus Mueller. vol. secundum. 8. Paris. 1848 ,

25. Fragmenta partim inedita Polybii, Dionysii Halicarnassensis, Polyaeni, Dexippi, Eusebii in Atho monte a Minoide Mina descripta edidit C. Mueller. - $18 \mathrm{~s}$.

26. Excerpta e Polybio, Diodoro, Dionysio Halicarnassensi 
atque Nicolao Damasceno, e magno imperatoris Porphyrogeniti

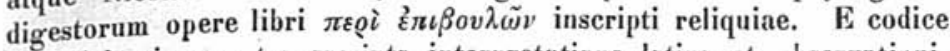
Escorialensi a se transscripta interpretatione latina et observationi-

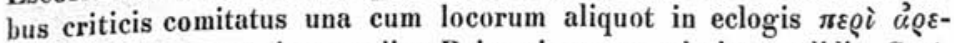
$\tau \tilde{\eta} \varsigma$ xui $x a x l a \varsigma$ ex ipso codice Peiresciano emendatione edidit $C$. $A$. L. Feder. p. I. Polybii, Diodori atque Dionysii fragm. 4. Darmstadii. Leske. 1840 . - $80 \mathrm{~s}$.

27. Excerpta e Polybio, Diodoro, Dionysio Halicarnassensi atque Nicolao Damasceno e magno imperatoris Constantini Porphyrogeniti

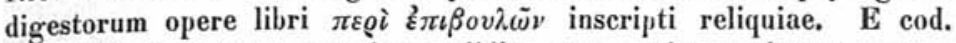
Escorialeusi a se transcripta edidit cum notis maximam partem criticis C. A. L. Feder. p. 1. Polybii, Diodori atque Dionysii fragmenta cum Nicolai XXV prioribus. 4. Darmstadii. Leske. 1849. - $134 \mathrm{~s}$.

28. C. Peter, das verhältniss des Livius zu Dionysius von Halicarnass zu einander und zu den alten annalisten. 4. Anclam. 1853.

29. C. G. Cobet, variae lectiones, quibus continentur observationes criticae in scriptores Graecos. 8. Lugd. Bat. Brill. 1854 ; ed. 2.1873.

30. De Dionysii Halicarnassensis quibusdam locis emendandis dissert.-philologica, quam . . scripsit J. J. Schmitz. 8. Bonn. 1854. - $26 \mathrm{~s}$.

31. Exercitationes criticae in Dionysii Halicarnassensis antiquitates romanas. Commentatio .. quam .. scripsit C. Sclnelle. 8. Bonn. 1854. - 36 s.

32. Carolus Sintenis, Emendationum Dionysiacarum specimen I. ad virum clarissimum F. Ritschelium, professorem Bonnensem. 4. Servestae Anhaltinorum. 1856. - 31 s.

33. K. W. Krïger, index zur griechischen sprachlehre, p. 282.

34. C. G. Cobet, novae lectiones, quibus continentur observationes criticae in scriptores graecos. 8. Lugd. Batav. Brill. 1858.

35. De Dionysii Halicarnassensis antiquitatum auctoribus latinis. Dissertatio philologica, quam . . scripsit Ad. Kiessling. 8. Lips. Teubner. 1858. - $43 \mathrm{~s}$.

36. H. Fischer, de aliquot locis antiquitatum Romanarum Dionysii Halicarnassensis. 4. Meiningen. 1858.

37. Mnemosyne, bibliotheca philologica. Scripserunt et collegerunt C. G. Cobet, T. J. Habertsma etc. vol, nonum. 8. Lugd. Batav. 1860 , s. $315-323$.

Aus verschiedenen gründen scheint uns der gegenwärtige zeitpunkt geeignet einen prüfenden rückblick auf die leistungen für Dionysius von Halikarnass zu werfen, für den wir bekanntlich erst seit sechs jahren eine kritische ausgabe von Ad. Kiessling besitzen. Jetzt soll, wie wir aus den mitteilungen der verlagsbuchhandlung B. G. Teubner in Leipzig (nr. 1. 1876) entnehmen, in der bibliotheca scriptorum graecorum et romanorum Teubneriana von dem- 
selben verfasser eine $z w e i t e$ und doch jedenfalls vielfach verbesserte auflage erscheinen, wonach also ein früherer plan eine grössere ausgabe der archaeologie des Dionysios mit vollständigem kritischen apparate zu veranstalten als aufgegeben zu betrachten ist. Ein jahresbericht nun, der es unternimmt alle bisherigen leistungen für diesen schriftsteller zu besprechen, muss naturgemäss in zwei sehr ungleiche teile zerfallen. Denn während der erste die ganze zeit bis zum jahre 1860 zu umfassen haben wird, in welchem der erste band der Kiessling'schen ausgabe erschien, gehört dem zweiten theile die litteratur von diesem zeitpunkte bis auf den heutigen tag an. Bei dieser eintheilung ist dann aber ferner auch nocb der umstand in betracht zu ziehen, dass von dem erscheinen des ersten bandes der genannten ausgabe bis zu dem des vierten und letzten zehn jahre vergingen, so dass wir also erst, wie gesagt, seit 1870 für das ganze werk unseres Dionysios einen den anforderungen der heutigen zeit genügenden text besitzen. Doch auch die erste bei weitem grössere periode zerfällt für sich wiederum in zwei abschnitte, die durch das jahr 1838 gebildet werden; damals nemlich gab bekanntlich Fr. Ritschl sein erstes programm über Dionysius heraus, dem später zwei andere nachfolgten und leitete dadurch die beschäftigung mit diesem schriftsteller in diejenige bahn, in der sie von da an geblieben ist.

Wenn nun zu dieser gegebenen eintheilung nach jabren unsere nachfolgende besprechung dem umfange und der genauigkeit nach im umgekehrten verhältniss stehen wird, so wird das niemand wunder nehmen; sind doch alle leistungen bis 1860 resp. $1870 \mathrm{mehr}$ oder minder bei Kiessling selbst zur verwendung gekommen.

Indem ich in bezug auf diejenige litteratur, die vom jahre 1480, wo die lateinische übersetzung des Florentiners Lapus Biragus herauskam, bis 1774 erschienen ist, auf Fabricius Biblioth. graec. vol. IV. p. 386 ed. Harl., sowie auf die artikel über Dionysios in der Realencyclopädie der classischen altertumswissenschaft von A. Pauly II. p. 1082 ff., und von Ersch und Gruber I. s. 25 p. 332 ff. verweise, beginne ich meine betrachtung mit $1774 \mathrm{~d}$.h. also demjenigen jahre, in welchem die unter Jo. Jac. Reiske's namen gehende ausgabe (nr. 1) ihren anfang nahm. Wie schon der titel anzeigt, sind in dieser ausgabe sämmtliche schriften des Dionys enthalten, nicht nur die antiquitäten in tom. I-IV auch die von Ursinus und Valesius veröffentlichten fragmente sind tom. IV p. $2309 \mathrm{ff}$. zum abdrucke gekommen, - sondern auch die rhetorischen schriften in tom. V und VI. Ausser einem index verborum et formularum quae in $\boldsymbol{D}$. $\boldsymbol{H}$. ant. rom. und einem ebensolchen quae in rhetoricis $\boldsymbol{D}$. $\boldsymbol{H}$. operibus visae sunt memorabiliores finden wir für beide werke noch einen index rerum notatu digniorum, sämmtlich am schlusse des VI. bandes. Der IV. band endlich enthält in seiner zweiten hälfte noch eine zusammenstellung der leges 
regiae et leges decemvirales Iusti Lipsii opera studiose collectae und Henrici Dodwelli chronologia graeco-romana pro hypothesibus Dionysii Halicarnassei mit den notae ad chronologiam.

Zwar ist es hinlänglich bekannt, kann aber nicht oft genug, wie ich glaube, betont werden, dass der antheil, welchen Reiske an dieser leipziger ausgabe hat, nur ein sehr geringer ist. Nachdem nemlich 1704 zu Oxford Hudson's ausgabe erschienen war und durch die lesarten des codex Vaticanus oder Urbinas aufsehen erregt hatte, beabsichtigte die genannte leipziger buchhandlung einen abdruck derselben zu veranstalten. Das ungünstige urteil Reiske's über jene mehr durch äussere schönheit, als durch innern werth ausgezeichnete englische ausgabe (vgl. Reiske tom. I praef. p. X) veranlasste den leiter der buchhandlung sich an Reiske zu wenden, und seine hülfe bei dem besagten unternehmen zu beanspruchen. Daraufhin nahm sich nun Reiske der sache in der weise an, dass man ihm die bogen vor dem abdrucke zur revision zuschickte, die er durchsah, sie von den vorhandenen zahlreichen druckfehlern der Hudson'schen ausgabe reinigte und die schlechtere vulgata durch die bessern lesarten des Vaticanus ersetzte, vgl. p. XII. Aber auch diese thätigkeit Reiske's nahm erst von p. 479 ihren anfang, da der druck bereits soweit gefördert war, als man sich an ihn wendete. Der eigentliche und unschätzbare werth dieser nun unter seinem namen gehenden ausgabe beruht demnach nicht sowohl im texte, als vielmehr in den scharfsinnigen jedem bande angehängten anmerkungen. Mehr über diese allgemein bekannte, bis 1870 vorwiegend gebrauchte, auch heute noch äusserst brauchbare ausgabe zu sagen, scheint mir überflüssig. - Dringend zu wünschen wäre es übrigens, dass jeder, der sich mit Dionys beschäftigt, doch wenigstens auch vorher, ehe er eine schlechte oder gute verbesserung vorschlägt, in den text und jedenfalls auch in die anmerkungen von Reiske hineinsehen möchte; es ist geradezu unglaublich, wie neuere kritiker diese erste anforderung, die man an sie stellen muss, vernachlässigen und dann als $\mathrm{ihr}$ eigenthum das ausgeben, was hundert jahre vor ihnen Reiske gefunden hat.

Wenn ich vorhin Reiske's ausgabe die bis 1870 vorwiegend gebrauchte nannte, so war mir dabei wohl bekannt, dass inzwischen noch eine stereotypische ausgabe (nr. 2) der sämmtlichen werke erschienen war. Da aber in dieser nur wieder der entstellte text abgedruckt wurde und weder die bessern lesarten der handschriften noch die so oft glänzenden änderungen Reiske's die gebührende beachtung fanden, so kaun diese ausgabe nicht weiter in betracht kommen.

Doch muss ich noch einmal zu den erscheinungen des vorigen jahrhunderts zurückkehren, da David Christian Grimm (nr. 3) jedenfalls nicht unerwähnt bleiben darf. Abgesehen nemlich davon, dass durch genauere kenntniss der handschriftlichen überlieferung 
eine reihe von seinen änderungen hinfällig geworden ist, finden sich hin und wieder auch heute noch brauchbare vorschläge, die, wie es ja so manchmal geht, von andern späterhin nicht beachtet, zum zweiten und dritten male wiederholt worden sind. So urtheilt Grimm fasc. II entschieden richtig über I, 69 (84, 23 K.), wenn er die worte iò $A$ A $v \varepsilon i \alpha \nu$ einem glossator zuweist. Wir finden diese ansicht ohne Grimm zu nennen von H.Sauppe Gött. gelehrt. anzeigen 1861 bd. III p. 1846 wiederholt. Ebenso weiss Ad. Kiessling, wie es scheint, nichts von dem vorschlage Grimms (fasc. IV) zu II, $47(170,19)$, woselbst wir in seiner ausgabe noch folgendes lesen:

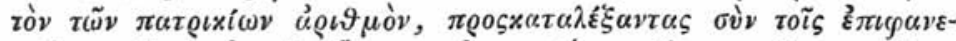

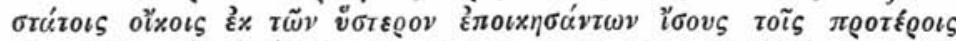

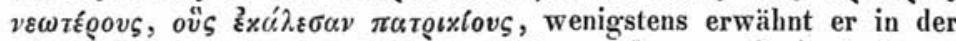

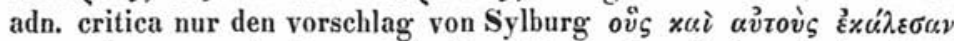

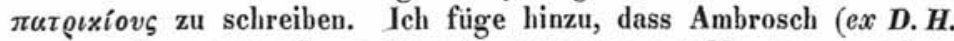
a. $r$. capita quae ad res Romuli pertinent $e$ codd. mss. emendata Vratisl. 1844) diese änderung für vollkommen sicher erklärt ebenfalls ohne auf Grimm rücksiclit zu nehmen, wogegen Kiessling später (zur kritik der röm. arch. des D. v. H. Basel. 1868. p. 8)

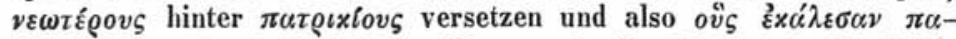

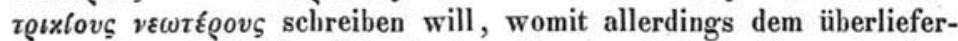
ten unsinn abgeholfen ist. Doch will mir scheinen, dass wir diese umstellung nicht brauchen, sondern sehr wohl mit Grimm nur die

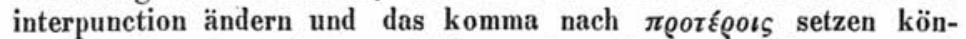

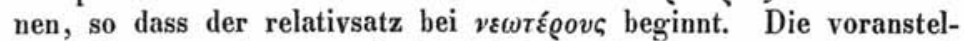
lung dieses wortes vor ov ${ }_{\zeta}$ ist dabei durch den gegensatz $\pi \varrho \circ \tau \dot{-}-$

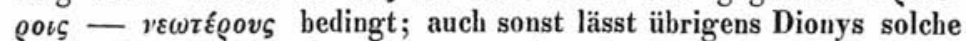
wortstellung nicht selten eintreten wie z. b. V, $75(206,9)$ ôv $\delta \xi$

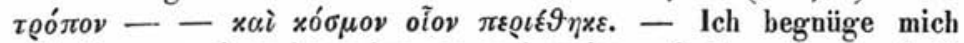
mit diesen wenigen bemerkungen und weise auf die grössere schrift desselben verfassers hin. In diesem buche (nr. 4) giebt Grimm einen auszug alles dessen aus Dionys, was sich auf verfassung, staats- und religionswesen des römischen staates bezieht, wobei er die reihenfolge der bücher einhält. Die unter den text, der nach Reiske gegeben wird, gesetzten anmerkungen sind theils erklärender art, theils geben sie die abweichenden lesarten und vorschläge anderer an. Am schlusse des buches findet sich ein brauchbarer index graecitatis.

Nachdem noch die schrift von J. Sam. Horstig (nr. 5) erschienen war, die mir unbekannt geblieben ist, ruhte die beschäftigung mit Dionysius bis zum jahre 1816, in welchem A. Mai die von ibm gefundenen fragmente herausgab (nr.6). Während die frühern ausgaben des Dionys (Reiske tom. IV. p. 2309-2365) nur diejenigen excerpte aus den verloren gegangenen büchern XII - XX enthielten, welche aus der bekannten excerptensamm- 
lung des Constantinus Porphyrogeneta unter dem titel exhoyui

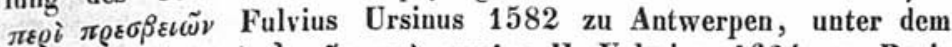

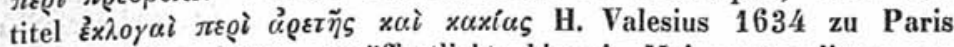
herausgegeben hatte, veröffentlichte hier A. Mai ausser diesen excerpten noch dasjenige, was er selbst auf der Ambrosianischen bibliothek zu Mailand in zwei papiernen handschriften (cod. Q s.XIV und cod. A s. XV) gefunden hatte. Zwar enthalten diese handschriften mehr als nur excerpte aus den uns verloren gegangenen büchern, allein A. Mai glaubte die aus den erhaltenen ihres geringen umfanges wegen weglassen zu dürfen. Dieses nun so aus verschiedenen stiucken zusammengesetzte buch erschien zuerst in gross quart in uncialbuchstaben gedruckt prachtvoll ausgestattet 1816 zu Mailand, ein jahr später zu Frankfurt als abdruck. Wenn auch die ansicht von A. Mai in diesem funde die von Dionysius selbst angefertigte epitome seines grossen werkes zu besitzen sehr bald durch Ciampi und Struve widerlegt wurde, so behielten deshalb natïrlich doch die neuen excerpte ihren werth.

Nicht lange nach dem erscheinen dieses so pomphaft angekündigten buches A. Mai's bewies C. L. Struve (nr. 7), dass die von A. Mai in der vorrede zu seinem buche ausgesprochene vermuthung den bis dahin für verloren gehaltenen auszug des Dionysius gefunden zu haben ein unding sei; die beschaffenheit der entdeckten bruchstücke, die aus kurzen inhaltsanzeigen und notizen neben langen briefen und reden beständen, mache die annahme Mai's geradezu unmöglich. Im weitern zieht Struve daraus, dass der grösste theil der Mailändischen excerpte, wie er sie kurz nennt, für sich besteht und nicht das wiederholt, was sich in den andern befindet, vier stellen sind allerdings auszunehmen, wo das nicht der fall ist den schluss, dass die mailändischen excerpte nicht zu jener grossen sammlung von auszügen des Constantinus gehört haben, sondern eine für sich bestehende excerpten-sammlung ausmachen. - Nach dieser auseinandersetzung über das verhältniss der drei excerptensammlungen zu einander, behandelt Struve die zweite wichtige frage: geben sie uns diese abschnitte aus des Dionysius grösserm werk wörtlich wieder, oder ist nur der sinn, das historische, aus dem Dionysius geschöpft, die einkleidung aber und die sprache eigenthum der spätern, welche excerpirt haben? Nach Struve nun lassen sich die fragmente in drei verschiedene abtheilungen bringen: 1) kurze inhaltsanzeigen ganzer abschnitte, oder 2) längere weitläuftigere auszüge, aber immer doch auszüge noch mit verkürzung der worte und auslassung von ganzen sätzen, oder endlich 3) grössere wörtlich übertragene stellen. Abgesehen von nr.3, wo natürlich die, eigenen worte des Dionysius wiedergegeben werden, beweist Struve, dass wir auch in nr. 1 und 2 meistens Dionysius eigene worte haben. Nachdem derselbe dann noch kurz zwei schon vorher nur erbobene anklagen gegen Mai beweist, nemlich dass ihm genaue 
sprachkenntniss und vielleicht auch übung im lesen alter griechischer manuscripte fehle, lässt er eine reihe von verbesserungen zum Dionysius, buch XII - XX, folgen, von denen wir sehr viele im texte von Kiessling wiederfinden. Ich begnüge mich die stellen des XII. buches hier anzuführen, um einen einblick in die Struve'

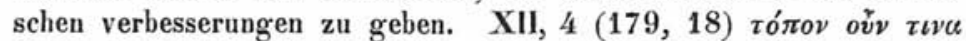
Aĩxov Mín

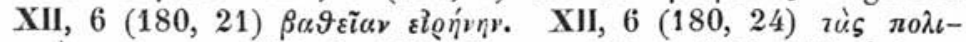

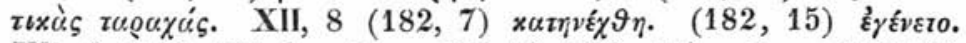

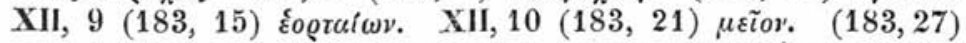

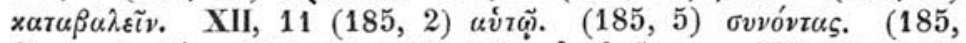

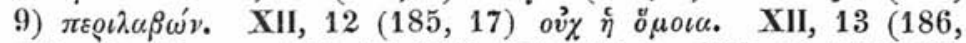

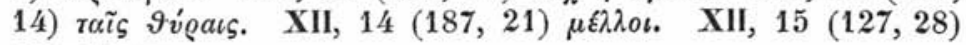
$\pi \varepsilon \rho\llcorner x \varepsilon i \mu \varepsilon v o v$.

Auch in den andern büchern ist die zahl der von Struve vorgeschlagenen und von Kiessling mit recht in den text aufgenommenen änderungen eine höchst bedeutende; wenn ihm natürlich auch nicht immer sofort die richtige verbesserung gelungen ist, so hat er doch wenigstens meistens dann das verdienst den sitz des fehlers richtig erkannt zu haben.

Bald jedoch sollte sich von neuem für Struve eine gelegenheit bieten auf die excerpte des Dionysius zurückzukommen. Im jahre 1827 nemlich erschien zu Rom der zweite band der Scriptorum veterum nova collectio e vaticanis codicibus edita ab Angelo Majo, enthaltend Historicorum graecorum partes novas. Hierin befand sich auch ein neuer titel der eclogen des Constantinus Porphyrogeneta, nemlich der $\pi \varepsilon \rho l \gamma v \omega \mu \tilde{\omega} v$. Obwohl sich nun im vaticanischen codex auszüge aus der archäologie des Dionys nicht befanden, nahm Mai dennoch in diesen zweiten band die schon früher einmal von ihm herausgegebenen fragmente (p. 465-526) auf, mit ausnahme allerdings der früher damit verbundenen Valesischen und Ursinischen excerpte. Dieser umstand bewog Struve eine abhandlung (nr. 8) zu schreiben, die ebenfalls mit recht viel aufsehen erregte: daher nur passend sie wie $\mathrm{nr} .7$ sich wieder abgedruckt findet in C. L. Struve opuscula selecta ed. J. Th. Struve, vol. I. p. $161-242$. Lipsiae 1854. 8. Seine frühere ansicht, dass sein aus verschiedenen excerpten zusammengestoppeltes buch einen theil einer von Dionysius selbst in fünf büchern verfertigten epitome seines werkes ausmache, hatte $\mathbf{A}$. Mai zwar in der vorrede seiner neuen ausgabe zurückgenommen, statt dessen aber drängt sich ihm, wie Struve Opusc. I p. 213 sich ausdrückt, nun ein neues phantom wieder auf, es seien nemlich diese excerpte ein integrirender theil des von ihm wieder aufgefundenen titels $\pi \varepsilon \rho i ~ \gamma \nu \omega \mu \tilde{\omega} v$. Weiterhin zeigt dann Struve klar und deutlich, dass die bewussten excerpte des Dionys dem genannten titel nicht angehört haben können. Ist doch nur sehr 
weniges darin gnomisch zu nennen; schlachten, zweikämpfe, belagerungen, geographische notizen, gründungen von städten u. dgl. machen den hauptinhalt aus. Einem bestimmten titel jedoch die excerpte zuzuweisen ist Struve nicht im stande.

Nachdem noch Pl. F. Schulin (nr. 9) und Weissmann, (nr. 10) über werth wie leben des Dionysius gehandelt hatten, erschien ein jalır darauf das von mir schon oben erwähnte erste programm Ritschl's. Durch diese schrift, in welcher derselbe das prooemium der archäologie d. h. also buch I, c. 1-8 mit kritischem apparate und dèr lateinischen übersetzung des Lapus Biragus herausgab, woran sich von p. 16 eine besprechung der Dionysiushandschriften anschloss, kam endlich auch die kritik in diesem schriftsteller in das einzig richtige geleise. Damit beginnt eine neue periode für Dionysius und es ist nur natürlich, wenn von nun an die beschäftigung mit diesem schriftsteller eine rege wird. Im vereine mit J. Ath. Ambrosch, der bei einem längern aufenthalte in Italien den kritischen apparat für die archäologie gesammelt hatte, gedachte damals Fr. Ritschl dieselbe in der weise herauszugeben, dass e r den gereinigten text, Ambros ch die erklärenden anmerkungen besorgte. Leider ist dieser plan nie zur ausführung gekommen und eine reihe von abhandlungen beider männer ist alles, was aus ihrer beschäftigung mit Dionysius herrorgegangen ist. Diesem plane gemäss gab nun bald darauf auch A mbrosch sein erstes programm heraus (nr. 12): auf ein kurzes vorwort, in welchem derselbe über die benutzten handschriften spricht - im übrigen verweist er auf die genannte abhandlung von Ritschl - und einigen wenigen bemerkungen über die sachliche erklärung, folgen die 29 ersten capitel des zweiten buches. - Schon im folgenden jahre liess Ambrosch eine zweite abhandlung folgen (nr. 13), in der er, wie schon der titel anzeigt, diejenigen excerpte aus Dionys herausgab, welche dem $\mathbf{X}$. buche angehören und ebenfalls in den von A. Mai zu Mailand auf der ambrosianischen bibliothek gefundenen papierhandschriften $\mathbf{A}$ und $\mathbf{Q}$ enthalten sind, von $\mathbf{A}$. Mai selbst aber damals nicht veröffentlicht worden waren. Es erfüllte durch den abdruck dieser excerpte Ambrosch wenigstens zum theil den wunsch Struve's, den er in seiner ersten abhandlung (opusc. vol. I. p. 168) geäussert hatte; zugleich aber wird seine vermuthung, dass in den grössern, wie in den kleinern excerpten soweit es irgend anging, Dionysius eigene worte beibehalten sind, bestätigt. Durch die von Ambrosch p. 1-8 vorangeschickten bemerkungen über die beschaffenheit dieser eclogae, sowie durch den text p. 9-18 und die im drucke deutlich gemachten verschiedenheiten der excerpte und des ganzen werkes, erhalten wir ein deutliches bild der eclogae Ambrosianae.

Gleichzeitig erschien ferner zu Berlin die abhandlung von Busse (nr. 14), über die ein urtheil in Jahn's jahrb. f. phil. und 
paed. bd. 35 (1842) p. 105 also lautet, das ich mir hier kurz wiederzugeben erlaube, da mir die schrift selbst unbekannt geblieben ist: eine klare und umfassende untersuchung, hervorgerufen durch Niebuhrs zweifel an dem historischen werthe des Dionysius als geschichtsschreibers, und auf die widerlegung derselben, wie auf die berichtigung mehrerer ansichten Krüger's und Ulrici's über Dionysius gerichtet. In drei abschnitten verhandelt der verf. I) de vita et arte rhetorica Dionysii; II) de philosophia Dionysii; III) de historiae vi et natura, was der schwächste theil der untersuchung ist, weil des Dionysius geschichtswerk zu sehr von den gegenwärtig herrschenden gesichtspunkten der geschichtschreibung aus beurtheilt ist: weshalb auch die gegen Krüger und Ulrici gerichteten erörterungen kein gehöriges gewicht erlangen.

Zur geburtstagsfeier von Friedrich Wilhelm IV. $1842 \mathrm{gab}$ Ambrosch die festschrift heraus, welcher wiederum eine abhandlung über Dionysius (nr. 15) voranging, an welche sich von demselben vf. im folgenden jahre eine zweite abhandlung (nr. 16) anschloss. Im ersten programm geht Ambrosch von der frage aus, ob das bei Dionys so häufig wiederkehrende wort $\gamma \varepsilon v \varepsilon \alpha^{\prime}$ eine bestimmte anzahl von jahren bezeichne - nach Glareanus, Sylburg und andern soll $\gamma \varepsilon v \varepsilon \tilde{c}^{\prime}$ einen zeitraum von 27 jahren umfassen, wogegen H. Dodwell in seiner abhandlung de aetate Dionysii Halicarnassei

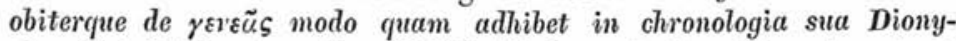
sias (bei Reiske tom. I. p. XLVI ff.) leugnet, dass Dionysius durch $\gamma \varepsilon v \varepsilon \dot{c}^{\prime}$ einen bestimmten zeitraum von jahren ausdrücken wollte ausgehend also von diesem worte $\gamma \varepsilon v \varepsilon \dot{a}$ prüft Ambrosch diejenigen stellen der archäologie, die dabei in betracht kommen, doch so, dass er sich hin und wieder nöthige abschweifungen erlaubt. Aus einer reihe von stellen, die Ambrosch \& 1-7 bespricht, kommt er \& $8 \mathrm{zu}$ dem schluss: haec omnia, quae attulimus, id videntur efficere, ut Dionysium vocabulo $\gamma \varepsilon v \varepsilon a ́$ spatium temporis certum idque annorum XXVII, si non ubique, at certe plus semel significare, non temere exislimemus, und geht zugleich zu solchen stellen über, die sich dieser annahme nicht fügen zu wollen scheinen \&̨ 8-11. Nach solchen vorbereitungen macht er sich \& 12 an eine stelle, über die er sich also äussert: locum si verba consideras, facillimum, sin sententiam in paucis difficilem; er meint damit III, 69 extr.,

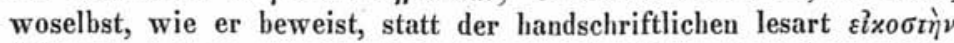

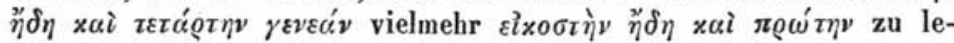
sen sei; hereingekommen aber ist der fehler durch die verwechselung der abschreiber von $\boldsymbol{A}$ und $\boldsymbol{A}$. Bei Kiessling findet sich in der adn. critica hierauf wunderbar genug keine rücksicht genommen. Anknüpfend an das gewonnene resultat bespricht endlich Ambrosch $\S 13$ noch solche stellen, welche sich auf das leben des Dionysius beziehen. Es schliesst derselbe seine eingehende und sorgfältige abhandlung, indem er in bezug auf die frage quid nobis de tem- 
pore antiquitatum tam conscriptarum quam editarum, atque de usu

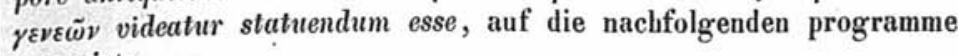
verweist.

Im anschlusse hieran handelt nun Ambrosch in dem bereits oben angeführten programm des jahres 1843 über die annahme der gelehrten, dass Dionys nicht mehr lange nach der herausgabe der antiquitäten gelebt haben könne, weil von allen schriften, auf die er in der archäologie ziemlich deutlich an verschiedenen stellen hindeute, uns keine spur erhalten sei, und weist dieselbe als unrichtig nach. Zur grundlage seiner untersuchung macht er dabei die

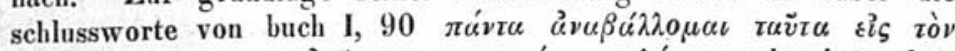

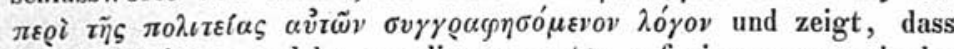
diejenigen irren, welche aus diesen worten auf ein neues werk des

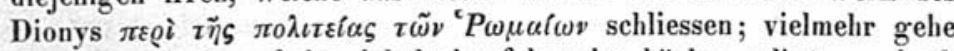
dieser ausdruck auf den inhalt der folgenden biicher selbst. - Auch für jedes der drei nächstfolgenden jahre ist je ein programm von Ambrosch zu verzeichnen: zuerst nr. 17, worin derselbe 26 capitel des II. buches, nemlich $30-56$ mit kritischen und erklärenden anmerkungen herausgab, so dass wir darin eine fortsetzung seines ersten 1840 veröffentlichten programms sehen können. Gleichsam das gegenstück dazu bildet nr. 18, worin nach kurzem vorwort in gleicher weise lib. II, 64-74 behandelt werden. In seinem letzten programm (nr. 19) endlich gab Ambrosch 29 capitel des ersten buches, nemlich lib. I, 9-38 heraus. Eine änderung zu den vorangehenden abhandlungen ist nur insofern eingetreten, als Ambrosch durch Hertzberg auf den cod. Elbingensis aufmerksam gemacht denselben hier zum ersten male mit benutzte.

Wahrscheinlich angeregt dnrch Ambrosch veröffentlichte J. K uschel eine abhandlung (nr. 20), welche mir nicht nälier bekannt geworden ist. Es sei mir deshalb erlaubt die worte Kiesslings aus seiner schrift de $D$. $H$. antiq. auctoribus latinis p. 5 herzusetzen, welche also lauten: neque multum profecimus Kuschelii docta et diligenti de primi antiquitatum libri fontibus dissertatione Vratislaviae $1842(?)$ edita, cum illius libri rationes tam sint singulares, ut inde fere nihil de relicuis adsequi possis. - Ausser diesen beiden arbeiten über Dionysius ist aus demselben jahre noch das zweite programm von Fr. Ritschl zu verzeichnen (nr. 21), in welchem derselbe nach einem kurzen vorwort $(1-4)$ den griechischen text und die lateinische ühersetzung des Lapus Biragus von buch I, 9 - 30 zugleich mit dem kritischen apparat herausgab.

Gerade ein jahr später erschien das dritte und letzte programm Ritschl's über Dionysius (nr. 22). Wenn wir hier auf diese für die kritische behandlung des Dionysius äusserst wichtigen abhandlungen Ritschl's nicht weiter eingehen, so geschieht es deshalb, weil wir es für besser halten die handschriftenfrage, wenn auch nur kurz, nachher im zusammenhange zu erörtern. Darum sei für 
jetzt auf Cobet (nr. 23) ebenfalls nur hingewiesen; wir werden später sehen, inwiefern auch dieses werk in einen jahresbericht über Dionysius gehört.

Das jahr 1848 brachte uns wiederum eine vermelirung der fragmente des Dionysius, indem Carl Müller in seinen Fragmenta historicorum graecorum bd. II. (nr. 24) zu den fragmenten von 72 historikern noch hinzufügte: bisher unedirte fragmente von Diodor, Polybius, Dionysius von Halikarnass aus einem codex Escorialensis p. VII-XLII. In der vorrede p. IV spricht C. Müller mit wenigen worten äber den codex Escorialensis, der wie wir erfahren, dem 16. jahrhundert angehört und ausser Aelians variae

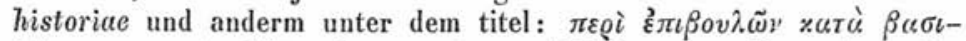

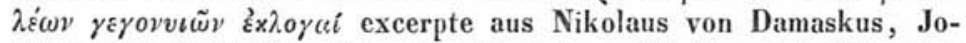
annes von Antiochia, Georgios Monachos, Diodor und Dionysius von Halikaruass enthält. Was nun die fragmente aus letzterem anbelangt, die uns ja natürlich einzig und allein hier angehen, so finden sich diese p. XXXI-XLII, und zwar sind sie buch $\mathbf{X I I}, \mathbf{X V}$ und $\mathbf{X X}$ entnommen. Schon früher übrigens hatte $\mathbf{C}$. Müller fragmente des Dionysius aus dem titel $\pi \varepsilon \rho i ~ \sigma \tau \rho \alpha \tau \eta \gamma \eta-$ $\mu \alpha \dot{\tau} \omega \nu$ herausgegeben und sie dem band II. der ausgabe des Flavius Josephus von W. Dindorf, Paris 1847, angefügt (nr. 25). Be-

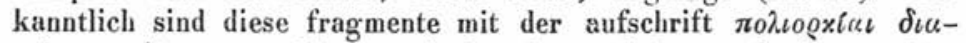
чó übrigens auch von G. Wescher unter dem titel Poliorcétique des Grecs etc. Paris 1867 veröffentlicht worden.

Gleichzeitig mit Müller gab auch C. Aug. L. Feder (nr. 26), die genannten fragmente heraus, der schon achtzehn jahre früher dieselben aufgefunden, damals auch genau abgeschrieben, die bekanntmachung derselben aber immer von einem jahre zum andern verschoben hatte. Darauf gab fast unter gleichem titel derselbe vf. die abhandlung nr. 27 heraus, wozu ferner noch eine dritte kommt, die mir unbekannt geblieben ist; sie erschien 1855. Ich muss hier darauf verzichten eingehender über die verbesserungen und vorschläge von Müller, Feder und andern zu handeln, und bemerke nur, dass auch sie wie Struve an nicht wenigen stellen zur verbesserung des oftmals arg entstellten textes der verloren gegangenen bücher beigetragen haben.

Nur im vorübergehen sei auf C. Peter (ur. 28) aufmerksam gemacht, da ich das programm nicht habe erlangen können; (es ist dem vernehmen nach dem vf. selbst nicht mehr möglich, ein exemplar anzuschaffen) doch wird sein inhalt wenigstens zum theil vom vf. selbst in seinem aufsatze: Dionysius von Halikarnass und Livius (Rhein. mus. XXIX. p. 532) angedeutet.

Das jahr 1854 brachte drei mir bekannt gewordene arbeiten über Dionysius welche sich sämmtlich mit texteskritik beschäftigen. Ich meine die von Cobet (nr. 29), von Schmitz (nr. 30) und 
Schnelle (nr. 31). Cobet nimmt an zahlreichen stellen seines buches auch auf unsern historiker rücksicht und verbessert mit glücklicher hand den oftmals schlimm entstellten text. Das ist z.b. der fall p. 6, wo er das handschriftlich überlieferte $\varepsilon v ่ \tau v \chi \tilde{\omega} \varsigma$

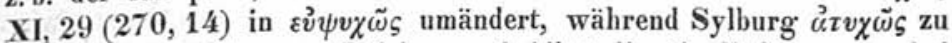
schreiben geneigt war; Reiske vertheidigt die überlieferung, wobei er dahin gestellt sein lässt, ob es mit $\ddot{\alpha} \pi \circ \vartheta \alpha \nu \varepsilon \tilde{\imath} \nu$ oder $\ddot{u} \gamma \omega \nu \iota \zeta_{0} \mu \xi-$ yous zu verbinden ist. L. Kayser, der Cobet's schrift in Fleck. jahrb. bd. 73 (1856) angezeigt hat, nennt p. 166 diese änderung Cobet's nicht gelungen, sondern verlangt mit Reiske (muss heissen Sylburg) $\ddot{\imath} \tau v \chi \widetilde{\omega} \varsigma$, das das vorhergehende cap. verlange. Kiessling hingegen hat $\varepsilon \dot{v} \psi v \chi \tilde{\omega} \varsigma$ in den text aufgenommen, nur durfte er in

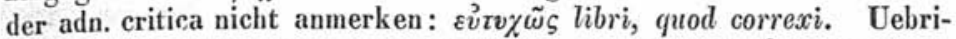

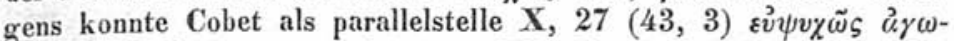

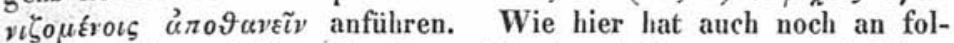
genden andern stellen Kiessling sich eine änderung zugeschrieben, die doch zehn jahre früher Cobet gemacht hat; demnach wird sein

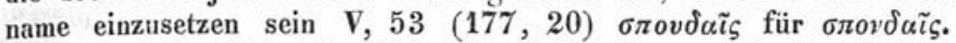

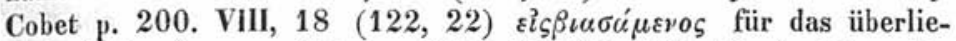

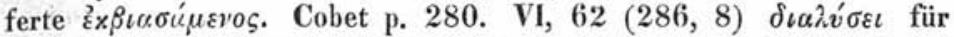
$\delta\left\llcorner\alpha \lambda v^{\varepsilon} \varepsilon\right.$. Cobet p. 362, der auch $\delta \iota \alpha \chi \varepsilon \tilde{\imath}$ im fut. verlangt; ob mit recht bleibt dahingestellt; Kayser billigt es p. 166. Wälırend hier also Cobet das verdienst der verbesserung gebührt, hat er anderswo selbst änderungen vorgeschlagen und sich zugeschrieben, die Sylburg, Reiske und andern zukommen: $\mathbf{X}, 1(2,3)$ war tôิ

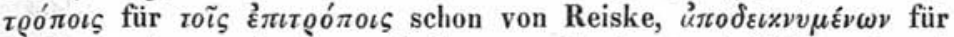
$\varepsilon \pi \iota \delta \varepsilon \iota x \nu v \mu \varepsilon \dot{v} \omega \nu$ schon von Sylburg nach dem cod. Urb. verlangt, und steht bereits bei Reiske. Cobet 283. X, $44(67,14) \varepsilon_{\xi} \xi-$

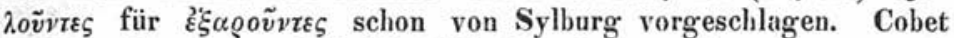

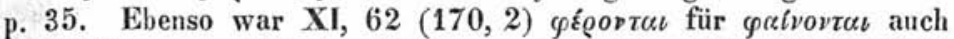
von Sylburg vor Cobet p. 121 angeraten, wie endlich III, 41 (279, $3)$ à $\lambda \dot{\alpha} \varsigma$ für $\ddot{\alpha} \lambda \lambda \alpha \varsigma$. Cobet 365 ; Kayser spendet ihm trotzdem für letztere änderung noch ganz besonderes lob. Mit recht hat Kiessling mit erwähnung seines namens aufgenommen VIII, $51(166,2)$

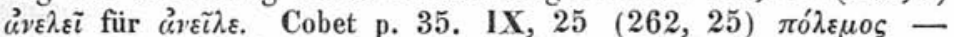

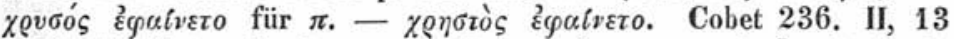
$(132,1)$ bieten die hs. ausser B nach $\ddot{\pi} \pi \alpha \nu \tau \varepsilon \varsigma$ noch $\delta$ xui of $v \tilde{v} v$ $\boldsymbol{x} \alpha \tau \hat{\varepsilon} \sigma \tau \eta \sigma \alpha \nu^{\prime}$, was Cobet 371 entfernt wissen wollte. Nicht anders verhält es sich mit den eingeschobenen worten VIII, $49(163,2$ ')

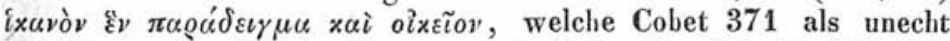
bezeichnet. - Nicht aufgenommen hat Kiessling IX, $47(294,19)$

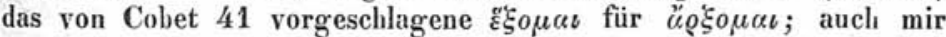
scheint eine änderung unnötig. Anführen konnte Kiessling in der adn. crit. zu VIII, $26(132,26)$, dass Cobet 298 die überlieferten

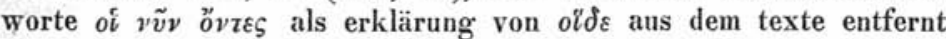

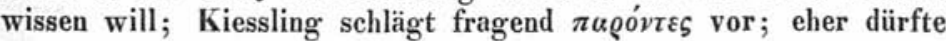


Cobet recht haben. - Die beiden andern schriften dieses jahres, nr. 30. 31, sind Bonner doctordissertationen durch Ritschl hervorgerufen. Schmitz behandelt in seiner kleinen schrift eine anzahl von stellen aus den ersten sechs büchern, wozu ihm Ritschl mit bekannter freundlichkeit die collationen vom cod. Urbinas und Chisianus zur einsicht gab. Von den angeführten vorschlägen, die er zur textesänderung macht, hat Kiessling eine kleine zahl späterhin

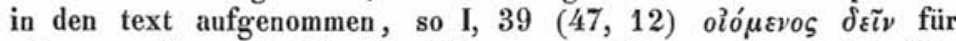

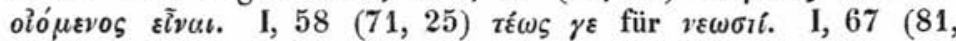

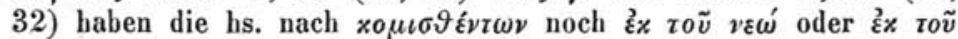

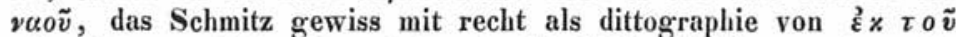

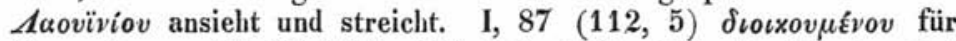

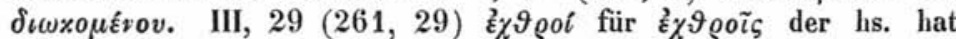
aufnahme gefunden. VI, $44(264,10)$ gehört die entfernung des $\eta$ Schmitz, nicht Kiessling an, der in der adn. crit. sechusi hinzufügt. -

Auch Schnelle (nr. 31) beschränkt sich auf die gleichen sechs bücher, wobei er ebenfalls Ritschl's collationen der hs. benutzen konnte; seine schrift zerfällt in vier capitel: 1) de simplicibus librariorum erroribus; 2) de glossematis; 3) de lacunis; 4) de transpositionibus. - Wenn auch das urtheil, das C. Sintenis (Zarncke's centralblatt 1865 p. 743) in den worten: selbst des herrn Schnelle zum theil höchst wundersame kritische versuche zu verzeichnen hat der herausgeber (Kiessling) nicht verschmäht, ein hartes ist, so ist es doch gerecht, und zum grössten teile wird die anführung der vorschläge von Schnelle künftighin auch in der adn. crit. unterbleiben können, geschweige denn dass ihnen im texte ein platz einzuräumen wäre. Nur sehr wenigen dürfte diese ehre zu theil werden. III, $16(235,1)$ nimmt Schnelle mit recht an ó $\mu o-$

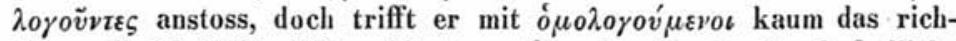
tige. $V, 36(155,15)$ schlägt er für das in den bs. befindliche

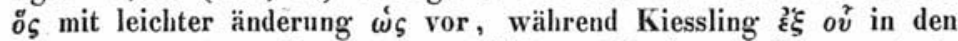
text setzt, später (Basl. progr. 1868 p. 15) o $\varsigma$ aus $\vdots \vartheta \varepsilon v$ verderbt sein lässt, wogegen $L$. Kayser in der recension dieses programms (Heidelberg. jahrb. 1868 p. 678) ö $s$ ohne ersatz gestrichen wissen will. Mir scheint $\omega \varsigma$ die einfachste änderung mit einem wenigstens erträglichen sinne. Unzweifelhaft richtig ist von Schnelle IV, $79(103,18) \quad \varepsilon \times \times o \mu \iota \delta \tilde{\eta} \varsigma$ hergestellt. V, $46(167,10)$ hat derselbe so weit recht, als ov vor $\mu \iota x \rho a ́ s$ fehlt; nur ist nicht xov zu schreiben; ebendaselbst hat Kiessling $(167,30) \pi 0 \lambda \lambda \tilde{\omega}$

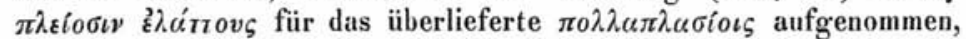
sowie $V, 53(177,30)$ auf seinen vorschlag av่toĩs geschrieben statt ovics.

In dem II. cap., das über glosseme handelt, finden sich neben sehr zahlreichen willkürlichkeiten nur wenig sichere beweise der

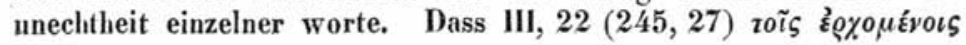


unecht sein muss, ist mir durch Schnelle's auseinandersetzung ebensowenig bewiesen, als ich III, $62(301,16)$ von der unechtheit

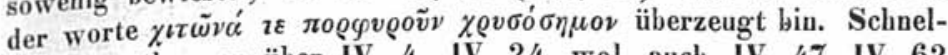
le's vermuthungen über IV, 4, IV, 24 wol auch IV, 47, IV, 62 werden wir künftighin auch in einer adn. critica gern entbehren. V, $17(133,15)$ ist Kiessling seinem vorschlage gefolgt und hat

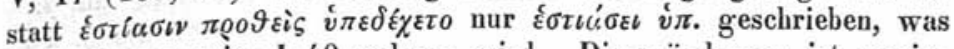
in ähnlicher weise I, 40 gelesen wird. Diese änderung ist wenigstens kein unding, wie so viele andere; aber nothwendig scheint sie mir auch nicht. Vielmehr ist wie II, 60 - Schnelle führt

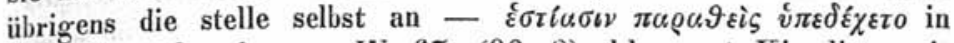
den text aufzunehmen. IV, $67(90,8)$ klammert Kiessling mit

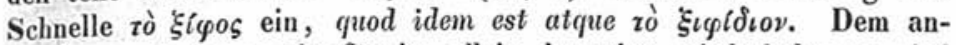
scheine nach ist es überflüssig, allein derartige wiederholungen sind gar nicht so selten, als man vielleicht glaubt; man vergleiche z. b.

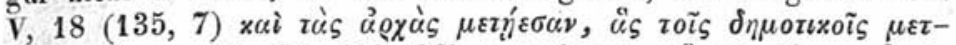

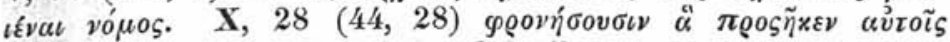

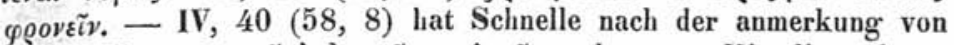

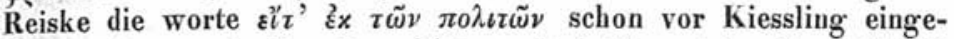
klammert, wonach die adn. critica zu berichtigen ist. Dass IV, 82 $(106,25)$ ảnó $\lambda \omega \lambda \alpha \varsigma$ ein glossem ist, wird Schnelle wohl niemandem so leicht glaublich machen. - Auch das III. cap., in welchem er lücken aufzudecken sucht, enthält hie und da richtige bemerkungen. I, 80 $(102,22)$ stimmt ihm Kiessling bei und nimmt nach oṽ $\alpha \nu$ den ausfall von einigen worten an. III, $62(302,29)$ will auch mir $\chi \varrho v \sigma o-$

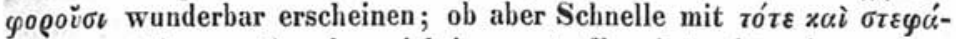

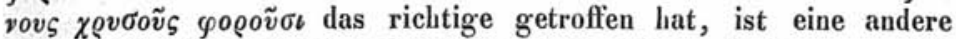
sache. IV, $60(82,6)$ tov่vavtiov vótıov ist wenigstens zum teil richtig gebessert; noch wahrscheinlicher jedoch urteilt Sintenis Hermes I (1866) p. 473 über diese worte. - Aus dem IV. cap. endlich ist $V, 2(113,31)$ mit recht das von Schnelle vorgeschlagene $\pi \varrho о \eta \gamma \varepsilon \tilde{\sigma} \vartheta \iota \iota$ für $\tilde{\eta} \gamma \varepsilon \tilde{\imath} \sigma \vartheta \leftrightarrow \iota$ von Kiessling aufgenommen. V, 29 (146, 28) und an andern stellen ist mir Schnelle's auseinandersetzung und wortumstellung unfassbar, so dass ich weiter darauf einzugehen verzichte.

Zu den ausgezeichnetsten leistungen aber, die ich für diesen ersten theil meiner besprechung zu verzeichnen habe, gehört die abhandlung von Carl Sintenis (nr. 32); auf eine kürzere einleitung, in welcher sich derselbe über Dionysius im allgemeinen, die verderbnisse des textes und die handschriftenfrage auslässt, folgt p. $9-31$ eine besprechung von mehr als 60 stellen; auch ihm hatte Ritschl die collationen des Urbinas und Chisianus zu verfügung gestellt. Eine eingehendere recension hat diese abhandlung von Sintenis durch Karl Schnelle in Fleck. jahrb. bd. 75 (1857) p. 377 erfahren, die von mir natürlich berücksichtigt werden wird; auch sei gleich hier bemerkt, dass Kiessling von Sintenis' vorschlägen mehr als ein drittel 
in den text aufgenommen hat: auch sind mehrere derselben später durch genauere vergleichung der handschriften bestätigt, wie z. b. VII, $12(16,31) \pi \alpha \varrho \eta \mu \varepsilon \lambda \eta \mu \varepsilon ́ v o \iota \varsigma$, für das auch bei Kiessling noch im texte sich vorfindende $\pi \alpha \varrho \eta \mu \varepsilon \lambda \eta \mu \varepsilon \varepsilon \omega \varsigma$. Sint. p. 11. I, $74(91,24)$

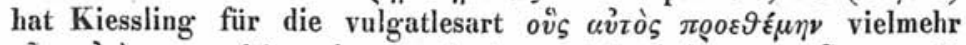

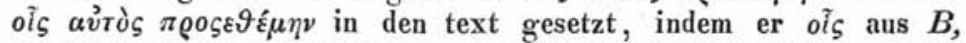
$\pi \varrho \circ \varepsilon \varepsilon \xi \mu \eta \eta$ aus $A$ aufnahm; dasselbe hat bereits Sintenis p. 16 verlangt. $V, 3(115,5)$ ist durch $B a$ die änderung von Sint.

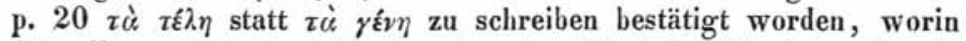
Schnelle p. 378 nicht beistimmte. X, $30(46,32)$ hat Sintenis

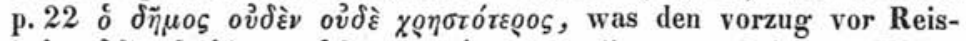

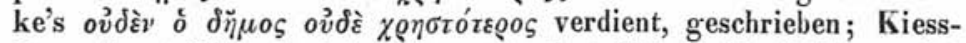
ling stimmt Sintenis bei; ob es handschriftliche überlieferung ist, ergiebt sich aus der adn. crit. nicht. Dasselbe ist IV, $11(17,28)$ der fall, woselbst Sintenis ov $\tau u^{3} \lambda \lambda o^{\prime} \tau \varrho \iota c$ schreibt p. 31 . Vorgeschlagen von Sintenis, gebilligt von Schnelle und mit recht in den text gesetzt von Kiessling sind folgende stellen: I, $31(37,23)$

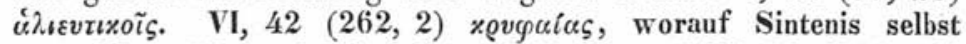
spec. III, p. 18 noch einmal zurückkommt. VIII, 88 (218, 26) die ergänzung von $\varepsilon \pi \iota x o v \rho l \iota$ nach $\varepsilon \pi \iota \circ \tilde{v} \sigma \alpha$, wenn nicht, wie Kiessling richtig hinzusetzt, das wort geradezu im participium darin

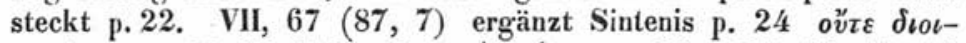

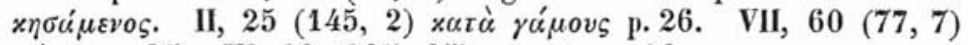

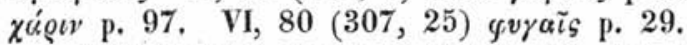

Während ich an diesen stellen mit den genannten derselben meinung bin, kann ich nicht I, $25(30,12)$ mit Sint. p. 10 und Bücheler $\tau o \tilde{v}$ vor $\pi \iota v \tau o ́ s$ streichen; erstens sehe ich die innere nothwendigkeit nicht ein, und zweitens verstösst die änderung gegen den sprachgebrauch des Dionysius (vgl. mein Aar. progr. p. 21); auch Ritschl und Kiessling haben es im texte gelassen. III, 14 $(232,14)$ zeigte es sich, dass die guten hs. vielmehr folgendes

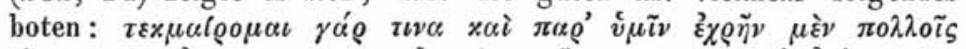

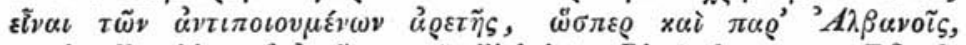
worin alles bis auf $\xi^{\prime} \varrho \tilde{\eta}^{\nu}$ verständlich ist. Die änderung von Pflugk

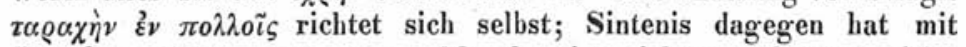
"้อıv $z v \pi_{0} \lambda \lambda_{0} \tilde{\tau}_{\varsigma}$, was er p. 18 als eine sichere änderung giebt, bei Schnelle, wie auch bei Kayser (Fleck. jahrb. 87 (1863) p. 11) grossen beifall geerntet. Kiessling nimmt die änderung zwar in den text auf, fügt aber in der adn. critica hinzu: quamquam ne sic quidem locum persanatum esse credo. Und daran tat er wohl. Paläographisch sehr leicht und für den sinn ganz vortrefflich scheint es mir, wenn wir aus EXPHNMEN vielmehr EYXEPEIAN EN

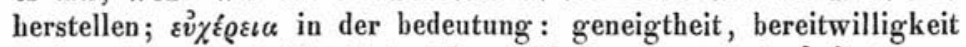

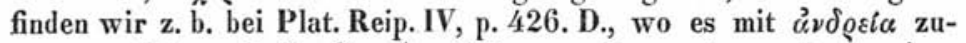

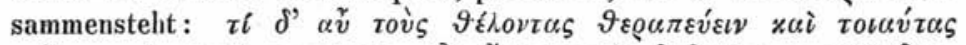

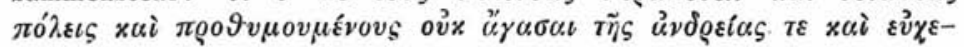




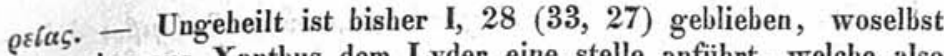
Dionysius aus Xanthus dem Lyder eine stelle anführt, welche also

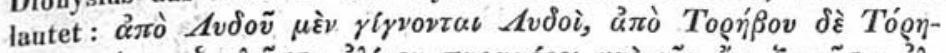

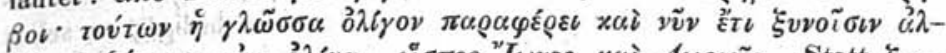

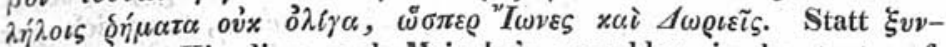
ov $\iota \nu$, was Kiessling nach Meineke's vorschlag in den text aufgenommen hat, findet sich in cod. A $\sigma \iota \lambda \lambda o v \sigma \iota v, \mathbf{B} \sigma i \lambda \lambda_{o v \sigma} \iota v$; Sintenis p. 15 empfieblt $\delta \mu o \lambda o$ yov $\sigma \iota v$. Dass beide vorschläge

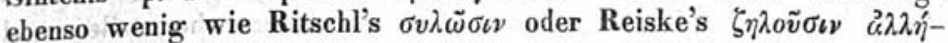

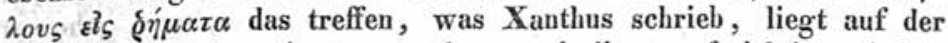
hand Ohne mit meiner vermuthung unbedingt auf richtige wiederherstellung anspruch zu machen, scheint sie mir wenigstens paläographisch so leicht und für den sinn angemessen, dass ich nicht umhin kann sie mitzutheilen. Indem ich nemlich davon ausgehe, dass das wort mit der präposition $\sigma v \nu$ anfangen und für $\ddot{a} \lambda \lambda \eta \hat{h} \lambda_{o v \varsigma}$ mit Sintenis und Meineke $\vec{u} \lambda \lambda \dot{r} \hat{\gamma}$ ovs geschrieben werden muss, bietet sich mir $C Y N A I A O Y C I N$ für CIAAOYCIN dar, so dass der sinn wäre: und auch jetzt stimmen sie in nicht wenigen worten überein; $\sigma v v \alpha \dot{\delta} \omega$ allerdings ohne accusativ lesen wir bei Dionysius selbst III, $26(256,2)$ und $X, 10(14,13)$; mit accusativ aber z. b. Plato Pol. IV p. 432 A. - II, $66(194,1)$ bietet B of

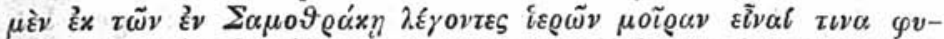

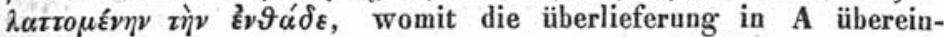
stimmt, nur dass für $\tau \iota v \alpha$ daselbst $\tau \tilde{\omega}$ Aivei $\alpha$ sich findet. Sintenis p. 17 schlug deshalb $\tau \iota v \alpha ~ \delta \iota \alpha \varphi v \lambda \alpha \tau \tau o \mu \varepsilon ́ v \eta \nu$ vor, was allerdings, wie Schnelle bemerkt (p. 380) wenig wahrscheinlichkeit hat; nur ist seine ansicht, dass hier entweder der ausfall eines wortes z. b.

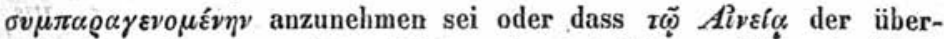
rest eines glossems sei um nichts wahrscheinlicher. Dass $\tau \tilde{\varphi}$ Alveía unsinn ist, scheint klar, ebenso klar wenigstens mir sein ursprung;

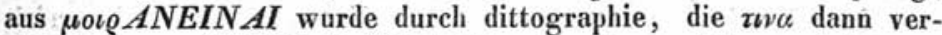
drängte, $\mu \circ \iota$ QNEIN AIAINEIAI, wozu dann später erst der artikel $\tau \tilde{\omega}$ hinzugefügt wurde (vgl. über diese stelle auch Ritschl Opusc. phil. I p. 536). - I, $24(29,29)$ war mit Sint. p. 10

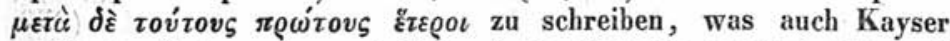
p. 11 im texte sehen möchte. Auch Sintenis vergisst hin und wieder in Sylburg's und Reiske's ausgabe hineinzuschauen, sonst würde er p. 9 bemerkt haben, dass schon Sylburg, dann auch

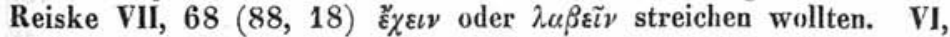
$56(278,11)$ rührt ì $\delta \ddot{\eta} \mu o \varsigma$ eigentlich schon von Sylburg her. Sint. p. 19. VI, $84(312,27)$ ist Kiessling meiner ansicht nach mit recht nicht der änderung von Sylburg gefolgt, der p. 25 für हlı vielmehr ไ̈ $\tau \varepsilon$ wollte, wenn ihr auch Schnelle p. 378 seinen beifall zollt. Ueber einige stellen, die Kiessling zuerst nach Sintenis verbessert hat, ist er später mit recht anderer ansicht geworden; so über II, $12(130,32)$ and II, $47(171,6)$. IX, 71

Philologus. XXXVI. bd. 1. 


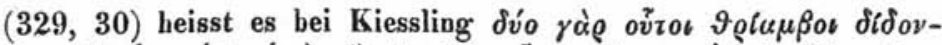

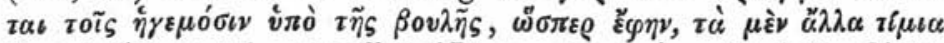

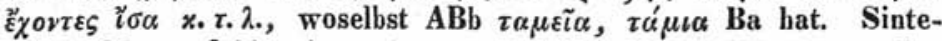
nis p. 28 empfiehlt $\tau \dot{c} \tau \iota \mu \tilde{\eta} s$, was Schnelle nicht billigt; er selbst verlangt mit berufung auf $\mathrm{V}, 47$ geradezu $\pi \alpha ́ v \tau \alpha$, was ich gar nicht für unmöglich halte; $\tau i \mu \iota \alpha$, was Kiessling sich zuschreibt, rührt von Sylburg her, wonach die adn. crit. zu ändern ist. -

Nach diesen bemerkungen über das erste programm von Sintenis breche ich hier $a b$, zumal da ich auf manche stellen und vorschläge noch später zu sprechen kommen muss, und gehe zu einigen änderungsvorschlägen von K. W. K r üg er über (nr. 33), die Kiessling ohne zweifel entgangen sind, da man dergleichen in einem index nicht sucht: in ihm hat Krüger unter der überschrift "ährenlese zu Dionysius von Halikarnass" zuerst zu b.I, dann unter "sporadisches" auch zu den andern änderungen vorgeschlagen, von denen wohl manche später eine stelle im texte verdienen. Dahin rechne ich: I, $8(10,10)$ ő worauf auch Sintenis p. 11 verfallen war. I, $37(44,16)$ vi $\lambda \eta_{\varsigma}$

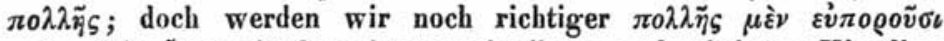
x $\alpha i x \alpha \lambda \tilde{\eta} \varsigma$ vँ $\eta \eta \zeta$ mit Casaubonus schreiben; vgl. übrigens Kiessling adn. crit. p. XVI und Sauppe Gött. gel. anz. 1861. p. 1852. I, 39

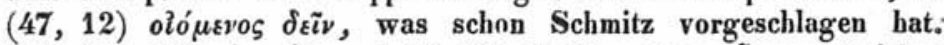

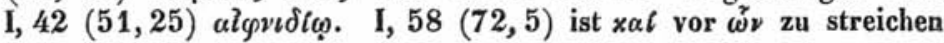
oder ${ }^{2} v$ x strichen. Aus der zweiten abtheilung hebe ich hervor: II, 73 $(203,26)$

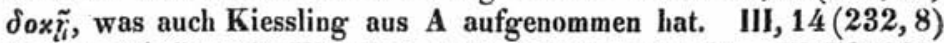

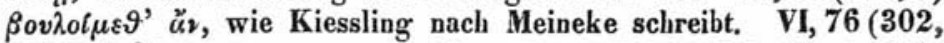
19) $\delta \grave{\eta} a ̉ \vartheta v \mu i \alpha \varsigma$ unter vergleichung von Thuk. VII, 55, 1. VII,

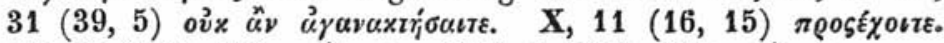
XI, $42(148,15) \pi \alpha v ́ \sigma o v \sigma i . \quad$ XVI, $2(222,22) x \omega i \grave{\alpha} \gamma \tilde{\eta} \zeta$.

In ähnlicher weise wie in den Variae lectiones behandelt C. G. Cobet in den aus der Mnemosyne wieder abgedruckten Novae lectiones (nr. 34) ebenfalls eine anzahl von stellen aus

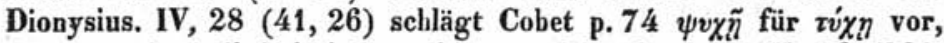
dessen nothwendigkeit ich wenigstens nicht einsehe. III, $19(238$,

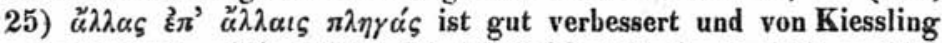
aufgenommen. Cob. p. 258. III, $59(299,16)$ है $\lambda \alpha \sigma \iota \nu$ Cob. p. 258.

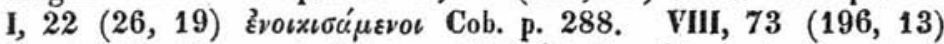

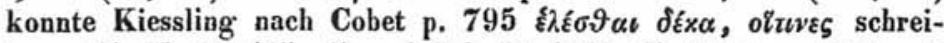
ben. 0 b X, $28(45,4)$ und XI, $52(159,6)$ Cobet p. 272 mit recht $\delta \varrho \tilde{\omega} v \tau \varepsilon \varsigma$ streicht, mag dahin gestellt bleiben. Denselben vorwurf, den ich bei besprechung der Variae lectiones Cobet machen musste, nicht immer auf vorschläge seiner vorgänger gebührende rücksicht zu nehmen, muss ich auch hier von neuem erheben. III,

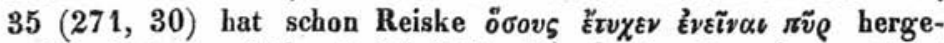

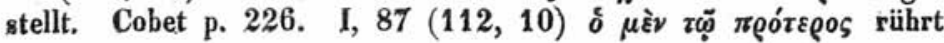




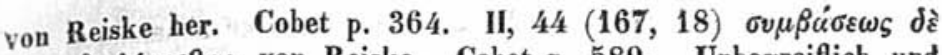

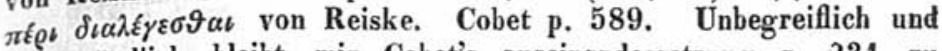
unverständlich bleibt mir Cobet's auseinandersetzung p. 231 zu

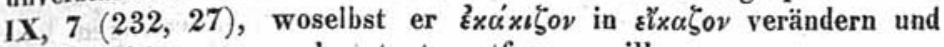

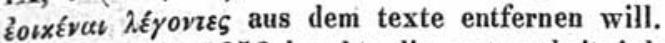

Das jahr 1858 brachte die erste arbeit Adolph Kiessling's (nr. 35), des nachherigen herausgebers der archäologie. Nach einem kurzen vorworte, in welchem derselbe die bisherigen leistungen für derartige quellenuntersuchungen im Dionysius anführt, behandelt er ausgehend von Dionys. I, 6; 7 die einzelnen schriftsteller. Den anfang macht Kiessling p. 7 mit Q. Fabius Pictor, den, wie er unter anführung der einschlägigen stellen zeigt, Dionysius nicht habe als quelle benutzen wollen, p. 13. Dasselbe gilt vom nächstfolgenden, nemlich dem Cincius Alimentus, der, wie es scheint, fast immer dasselbe wie Fabius berichtet und seine geschichtserzählung zum theil ihm entlehnt hat, p. 15. An diese beiden schliesst sich M. Porcius Cato an, der bekanntlich zuerst die einheimische d. h. römische sprache bei der geschichtsschreibung anwendet. Ihn stellt Dionys. I, 7 voran, als er seine quellen nennt, worauf dann Fabius Maximus, Valerius Antias und Licinius Macer als weitere gleichberechtigte gewährsmänner folgen; nicht verhält es sich ebenso mit

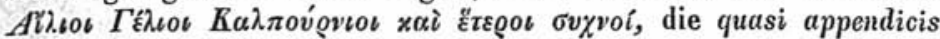
loco negligentius adiiciuntur neque temporum ratione servata neque nominibus plene positis, p. 16. Mit aufgabe der bisher innegehaltenen reihenfolge schiebt Kiessling zwischen Fabius Maximus p. 17 und Valerius Antias eine besprechung der historiker ein, welche Dionysius sonst noch bei der abfassung seines werkes verwendet hat; es sind dieses C. Sempronius Tuditanus, C. Acilius, Vennonius (L. Mallius nimmt er nur aus Varro); nicht ist Cassius Hemina verwendet; gezweifelt werden kann über Claudius Quadrigarius und gar nicht benutzt ist Livius, p. 18. Nach dieser abschweifung kehrt der vf. noch einmal zu M. Porcius Cato zurück, den Dionys mit und ohne anführung des namens wiederholentlich benutzt hat; schon das, dass auch Cato behauptet hatte, die Römer seien keine barbaren, sondern den Griechen verwandt, musste ihn dem Dionys empfehlen. Niemand aber war geeigneter als der nachfolgende, nemlich Valerius Antias, für die darstellungsweise unseres schriftstellers, p. 20-29. Reiche ausbeute gewährte auch Licinius Macer, p. 29 -33 , wogegen nicht sicher bestimmt werden kann, wie weit die benutzung des Q. Aelius Tubero sich erstreckt hat, p. 34. Mit Nipperdey nimmt Kiessling an, dass mit dem plural $T \xi \lambda_{\text {s. }}$ oc nur Gnaeus Gellius gemeint sei, an dem Dionys seiner breite wegen wohlgefallen gefunden zu haben scheint, p. 34. Der letzte in der reihe ist L. Calpurnius Piso, p. 35-38; bis zum schlusse der abhandlung spricht der vf. endlich über M. Terentius Varro, der ge- 
wiss mit vollem rechte ebenfalls eine stelle in der abhandlung verdient hat.

Auf eine eingehendere besprechung von Fischer's schrift (nr. 36) muss ich verzichten, da ich diese nicht habe erlangen können; doch sei bemerkt, dass sie Kiessling in der adnotatio critica hin und wieder anführt; so z. b. bd. II $p$. VII zu IV, $15(22,20)$, IV, $17(26,14)$ und sonst.

Ich schliesse den ersten theil meines bericlites mit Cobet's bemerkungen in der Mnemosyne (nr. 37), woselbst derselbe p. 315 - 23 verschiedene stellen aus der archäologie behandelt, welche sämmtlich den büchern I - IV entnommen sind. I, $67(82,23)$

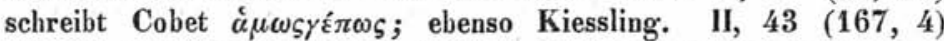
erkennt auch Cobet $x \alpha \vartheta^{\prime}$ ' $\psi \psi \eta \eta \hat{v}$ als die richtige lesart an, was mir (Aar. progr. 1874 p. 8) entgangen war und Tegge (quaest. de Dionysi H. usu praeposit. spec. I, p. 21) ebenfalls unbekannt geblieben ist. II, $44(167,18)$ hatte Cobet schon nov. lect. p. 589 in gleicher weise behandelt, ohne auch dort Reiske einzusehen. Dasselbe gilt von III, 35 Nov. lect. p. 226; von III, 41 Variae lect. p. 365 ; III, 11 zum theil Nov. lect. p. 281 . II, $55(179,10)$ ist $\tau o ́ n o v$, das Cobet aus dem texte entfernen wollte, späterhin von Kiessling (Basl. progr. p. 9) gut vertheidigt worden. III, 5 (215, 12) nimmt sich des von Cobet als unecht verdächtigten xo๙zov́ $\mu \varepsilon-$ vov Kiessling Basl. progr. p. 10 und nach ihm Tegge p. 34 anm.

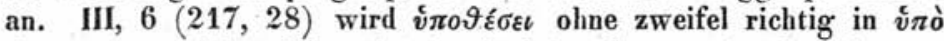
$\vartheta \varepsilon\{\alpha \varsigma$ verwandelt. Fine eingehende behandlung und mehrfache verbesserung findet III, 11, wo Reiske wiederum vernachlässigt wird.

Wie ich schon oben erwähnt habe, scheint es mir der einfachbeit wegen am besten über die handschriftliche grundlage, auf welcher die texteskritik des Dionysius beruhen muss, hier, ehe ich zum zweiten theile meines berichtes übergehe, kurz im zusammenhange zu handeln. Während Robert Stephanus bei der herausgabe des Dionysius 1576 den schlechten codex Parisinus, Sylburg noch ausserdem den um nicht vieles bessern cod. Venetus benutzte, stand Hudson allerdings eine collation des cod. Vaticanus oder Urbinas zu gebote, nur machte er nicht den gebrauch davon, der nötig gewesen wäre; Reiske endlich besass keine vergleichungen der handschriften, sondern nahm nur hin und wieder diejenigen lesarten des Vaticanus auf, die ihm unbedingt richtig erschienen. Dass unter solchen umständen in erster linie eine neue sorgfältige vergleichung und prüfung der vorhandenen handschriften des Dionysius erforderlich war, musste jedem, der sich mit ihm beschäftigen wollte, sofort klar sein. Das material wurde zuerst von Jul. Ath. Ambrosch bei einem aufenthalte in Italien gesammelt, wie uns Ritschl in der vorrede zu seinem ersten programm (1838) mittheilt, dann zum ersten male von letzterm ebenda in kritischer weise gesichtet. Ritschl's eingehende untersuchung erstreckt sich zuerst auf dieje- 
nigen handschriften im allgemeinen, welche von Lapus Biragus an zur verwendung gekommen waren; und zwar geht er dabei von den codices romani aus, welche sieben an zahl sind. Von dreien unter diesen, nemlich den beiden von Lapus und dem von Ursinus benutżten, die auch Sylburg in betracht zog, lässt sich wenig feststellen. Hudson standen die vergleichungen des vorzüglichen Vaticanus óder Urbinas n. 103 zu gebote, den er, da er nur zehn bücher umfasst, durch den Vaticanus n. 450 für buch XI ergänzte. Ambrosch endlich verschaffte sich vom Chisianus, einer handschrift des X. jahrhunderts, die ebenfalls nur zehn bücher enthält, und dem Vaticanus 1300 vergleichungen. An diese erörterung über diese römische handschriften reiht Ritschl die der codd. Veneti und des $\mathbf{P a -}$ risinus, der zuerst durch Rob. Stephanus ans licht gezogen worden ist; verschieden von diesem, für die kritisclie grundlage hin und wieder wichtig, ist der cod. Coislinianus n. 150, aus dem XV. jahrhundert stammend und auch das XI. buch umfassend. Nach dieser auseinandersetzung im allgemeinen geht Ritschl zur erörterung der frage über, wie die genannten handschriften zur verwerthung kommen sollen. Auszuscheiden sind zuerst die Veneti, die fast immer mit dem Urbinas gehen; auch die von R.Stephanus benutzte handschrift kann nicht weiter in betracht kommen gegenüber den beiden handschriften, die allen voranstehen, dem Chisianus und Urbinas. Auf der richtigen beurtheilung dieser beiden handschriften berubt, wie es Ritschl schon in diesem ersten programm (0pusc. phil. I, p. 484) in den worten: est autem hoc eo et religiosius et distinctius expendendum, Chisianusne an Urbinas in primo sit loco habendus, quo certius hac ratione fundamentum criticae operae in Dionysii antiquitatibus ponendae iacitur ausspricht, die texteskritik in Dionysius. Bei der untersuchung dieser schwierigen frage, über die man das einżelne bei Ritschl selbst nachlesen möge, kommt derselbe p. 484 zu dem resultat, dass die grössern vorzüge der Chisianus enthält, ohne im geringsten zu verkennen, dass derselbe andererseits oftmals hinter dem Urbinas zurücksteht; das enthalten deutlich genug die worte p. 486: verum non ita tamen Chisianus praestat, ot sine exceptione posthabendus sit Urbinas, wofür die belege folgen. Und in ähnlicher weise äussert sich Ritschl noch einmal, wenn er sagt p. 488: vix opus fuerit de summa norma critici in $D$. ant. negotii phuribus exponere. quam satis apparet hanc debere esse, ut a Chisiani parte stemus, quam diu possimus, nec tamen vereamur ad Urbinatis fidem confugere, ubicumque id ipsa ratio vel poscat, vel non numquam tantum suadeat. Für das zweite programm, in welchem Ritschl I, 9-30, wie wir sahen, mit kritischem apparat herausgab, standen ihm für cod. Chisianus $(A)$ neue vergleichungen von A. Fea und Emil Braun zu gebote, so wie eine neue collation des Urbinas, die ebenfalls Braun besorgt hatte. Es unterscheidet demnach in zweifelhaften fällen Ritschl zwischen Bb, der Braun'- 
schen und $\mathbf{B h}$, der Hudson'schen vergleichung des Urbinas. Auch für den cod. regius $\mathbf{D}$ und Coislinianus $\mathbf{C}$ hatte Ritschl neue collationen sich verschaffen können, so dass er im stande gewesen wäre auf grundlage dieses handschriftlichen apparats die antiquitäten herauszugeben. Doch das unternehmen, an dem Ambrosch, wie wir sahen, sich durch die sacherklärung betheiligen wollte, unterblieb.

Veranlasst durch C. G. C ob et $\mathrm{s}$ auseinandersetzung in seiner schrift oratio de arte interpretandi p. 134 über die vorzüglichkeit des cod. A und durch eine recension seines ersten programms dureh Sintenis, die für das Rhein. museum bestimmt war, aber nie zum abdruck gekommen ist, fühlte sich Ritschl veranlasst in seiner dritten oben angeführten abhandlung de codice Urbinate (nr. 22) die grossen vorzüge von B ins helle licht zu setzen. Was Ritschl mit diesem programm wollte, spricht er deutlich genug p. 22 (0pusc. phil. I, 537) gegen ende der abhandlung aus, wo er sagt: reputandum est autem non hoc nobis nunc agendum fuisse, ut vel Chisiani in multis partibus praestantiam vel Urbinatis in aliis pravitatem commonstraremus, quippe de qua et praestantia et pravitate nemo dubitasset, sed hoc potius, ut Urbinatis suo in genere excellentiam contra iniustas, ut nobis quidem visum, unum Chisianum admirantium obtrectationes defenderemus. Dass mit den auseinandersetzungen in dieser abhandlung die in der ersten über den cod. Chisianus aufgehoben sein sollten, hat Ritschl nie gewollt. - Durch eine mit Jac. Bernays zusammen angestellte sorgfältige prïfung des Urbinas kam Ritschl zu dem gleich am anfange der abhandlung ausgesprochenen urtheil, welches also lautet: deterioris generis melius exemphum Chisianus, deterius autem melioris generis repraesentare Urbinas existimandus est.

In eingehender untersuchung, die durch beispiele belegt wird, zeigt nun Ritschl, dass cod. B vor A bedeutende vorzïge darin besitzt, dass an vielen stellen durch ihn allein, manchmal auch in verbindung mit A lücken ausgefüllt werden, die sich in den andern handschriften finden. \& 2. Die lücken, die durch B ausgefüllt werden, bestehen theils in grössern sätzen, die, ohne den gedankengang zu beeinträchtigen, manchmal fehlen können, ferner in einzelnen oder mehreren worten. \& $3-5$. Verderbte lesart in $\mathbf{A}$ und in den andern handschriften ist oft die veranlassung zu späterer interpolation gewesen, wogegen $\mathbf{B}$ die echte lesart bewahrt hat. \& 6 . unglaublich viel lïcken haben die sogenannten homoeoteleuta hervorgerufen. \& 8. Schwer wiegt beim abschätzen des werthes einer handschrift selbstverständlich der fall, dass die lesart der handschrift mit dem citat in einem andern schriftsteller übereinstimmt. Dieser fall trifft öfters bei $\mathbf{B}$ zu, wenn man die lesarten in B mit denen im Eusebius, Georgius Syncellus etc. vergleicht. \& 9. Auch abgesehen von den lïcken und deren ergänzungen zeigt oft cod. B, wie Ritschl \& 11 an beispielen nachweist, grössere sorgfalt. Nicht 
unwichtig ist bei der beurteilung ferner das vorkommen oder vermeiden des hiatus. \& 12 . Zum schlusse \& 14 giebt uns Ritschl durch eine figur eine anschauliche übersicht von dem verhältniss, in welchem seiner ansicht nach die handschriften zu einander stehen.

Nach solchen auseinandersetzungen, denen sich auch Ambrosch und Sintenis spec. I, p. 8 anschloss, musste es natürlich auf Ritschl und andere einen mehr als eigenthümlichen eindruck machen, wenn der neue herausgeber der archäologie Ad. Kiessling bd. I, p. VI in folgenden worten: oum autem Chisiani libri antiquissimi auctoritas apud Ritschelium Ambrosiumque tantum valuerit ut ad eius praecipue fidem scriptoris verba exigi posse existimarent, $m i$ hi res longe aliter sese babere videtur. Urbinatis enim praestantia quamvis levioris cuiusdam interpolationis commaculata vestigiis ea est at emendationem totam fere ab hoc libro repetendam esse mihi persuasum sit, einen ganz neuen standpunkt einzunehmen glaubte. Es lassen sich diese worte eben nur dadurch erklären, dass Kiessling in unverzeihlicher nachlässigkeit jene abhandlung Ritschls de codice Urbinate Dionysii Halicarnassensis disputatio 1847 übersehen hat; dafür spricht allerdings auch der umstand, dass er p. VI nur das erste (1838) und zweite (1846) programm Ritschl's erwähnt. Es war natürlich, dass ihm Sintenis (Zarncke lit. centralblatt 1861 p. 836) und Ritschl (0pusc. philol. I, p. 517 anm.) dieses vorhielten. Bis zu einem gewissen grade weicht Kiessling freilich in der beurtheilung des cod. Urbinas von den genannten ab, darin nemlich, wogegen mit vollem rechte H. Sauppe (Gött. gelehrt. anเeig. 1861 p. $1841 \mathrm{ff}$.) ihm entgegengetreten ist, dass er B zur ausschliesslichen grundlage des textes gemacht hat, in der adn. critica also auch nur dasjenige anführt, was von der lesart in B abweicht (vgl. p. VII: in adnotatione quae sequitur critica id egi ut quae ab Urbinatis libri scriptura recederent accurate indicarem). Dass sehr vieles, was Sauppe in jener recension über die vorzüge des A vorbringt richtig ist, wird niemand leugnen können; - namentlich auffallend auch für einen, der nur einen flüchtigen blick in B hinein thut, sind die theils aus nachlässigkeit, theils aus absicht hervorgegangenen, immer aber willkürlichen änderungen in B, wofür Sauppe p. 1847 eine reiche anzahl von belegstellen anführt, stellen, an denen Kiessling selbst dem cod. B nicht gefolgt ist -; andererseits geht aber doch Sauppe zu weit, wenn er B eine höchst bedenkliche grundlage des textes nennt und verlangt, dass man bei der feststellung dessen, was Dionysius geschrieben haben soll, immer von dem texte des cod. A ausgehe und nur dann, wenn innere gründe für die fehlerhaftigkeit dieser überlieferung, für die vorziiglichkeit des in B vorhandenen sprechen, dies als das richtige anerkenne.

Seine schon in der ersten abhandlung p. 8 ausgesprochene ansicht hat Sintenis in der zweiten p. 1 und dritten p. 1 mit be- 
rücksichtigung der von Cobet, Kiessling und Sauppe geführten untersuchungen des weitern begründet und jeder besonnene und nicht voreingenommene kritiker muss meiner ansicht nach unbedingt beistimmen, wenn er spec. II, p. 1 sein urtheil in die worte zusammenfasst : ex coniuncto utriusque libri usu cauto et iusto quodam quasi temperamento instituto rem agendam esse und spec. III, 1 - at mihi quidem, uti nunc res est, cum suas uterque codex virtutes, sua vitia habeat, nihil magis videatur opus esse, quam subtili iudicio quod idoneo scriptoris usu adiutum singulas lectiones in disceptationem vocet perpensisque rationibus omnibus id constituat, quod Dionysium scripsisse probabile sit. $\mathbf{Z u}$ diesem standpunkte, den Ritschl und Sintenis einnehmen, habe ich mich schon früher (Observat. crit. in D. H. p. 291) bekannt und bin bis jetzt noch durch nichts veranlasst worden, denselben zu verlassen; im gegentheil meine fortgesetzte beschäftigung mit Dionysius hat mir gezeigt, dass dieser standpunkt der einzig richtige ist, wenn auch Tegge p. 2 sich neulich wieder der ansicht von Kiessling anschliesst.

Zum schlusse habe ich nur noch $\mathrm{zu}$ bemerken, dass die handschriftliche grundlage für buch XI eine andere ist und zwar auf dem Laurentianus (L) und Vaticanus (V), die beide dem XV. jahrbundert angehören, berubt. Ich verweise auf das, was Kiessling tom. IV p. XII. darüber äussert und füge nur noch hinzu, dass der zuerst von Ambrosch benutzte cod. Elbingensis, den auch ich verglichen habe, mit $L$ geht.

Zweiter abschnitt.

1. Dionysi Halicarnasensis antiquitatum romanarum quae supersunt recensuit Adolphus Kiessling. Vol. I. Lipsiae in aedibus B; G. Teubneri. MDCCCLX. p. XLVII u. 318. 8.

2. H. Sauppe, Göttingisch. gelehrt. anzeigen 1861. bd. III. stück 47 p. $1841-1865$.

3. C. S. s. (Carl Sintenis) liter. Centralblatt für Deutschland herausg. von Fr. Zarncke, jahrg. 1861 p. $835 \mathrm{ff}$.

4. Heidelberger jahrbücher der literatur, jahrg. 54. nr. 9. 1861 p. $128 \mathrm{ff}$.

5. L. Kayser Fleckeisen's jahrb. für kl. philologie bd. 87 (1863) p. 1- 11.

6. Carolus Sintenis emendationum Dionysiacarum specimen II. Servestae Anhaltinorum MDCCCLXI. p. 36. 4.

7. Literar. Centralblatt für Deutschland herausgeg. von Fr. Zarncke, jahrg. 1862 p. 557.

8. Aug. Meinelie miscellanea. Fleck. jahrb. f. kl. phil. bd. 87 (1863) p. $369 \mathrm{ff}$.

9. H. Sauppe zu den griech. historikern Philologus bd. XIX (1863) p. 148.

10. L. Urlichs zu Dionysius von Halikarnass I. Eos, süd- 
deutsche zeitschrift. für philologie und gymnasialwesen. Herausgeg. von L. Urlichs, B. Stark etc. I. jahrg. Würzburg 1864. p. 309. 324.

11. Dionysi Halicarnasensis antiquitatum romanarum quae supersunt recensuit Adolphus Kiessling. Vol. II. Lipsiae in aedibus B. G. Teubneri. MDCCCLXIV. p. XLV. u. 328. 8.

12. Literarisches Centralblatt für Deutschland herausg v. Fr. Zarncke. jahrg. 1865. p. $741 \mathrm{ff}$.

13. Heidelberger jahrbücher der literatur. jahrg. 58 (1865) p. 351 .

14. L. Kayser, Fleck. jahrb. für kl. philol. bd. 93 (1866) p. $35-47$.

15. Carolus Sintenis emendationum Dionysiacarum specimen III. Servestae Anhaltinorum MDCCCLXV (nicht MDCCCXIV) p. 29.4 .

16. C. Sintenis zur kritik des Dionysius von Halicarnass. Hermes, zeitschrift für kl. philol. Herausg. von E. Hübner. bd. I. Berlin 1866. p. 142. 471.

17. Friderici Ritschelii opuscula philologica. Vol. I ad litteras graecas spectantia. Lipsiae in aedibus B. G. Teubneri. MDCCCLXVl. p. $471-540$.

18. Dionysi Halicaruasensis antiquitatum romanarum quae supersunt recensuit Adolphus Kiessling. Vol. III. Lipsiae in aedibus B. G. Teubneri. MDCCCLXVII. p. XXXV. u. 329. 8.

19. Literarisches Centralblatt für Deutschland herausg. von Fr. Zarncke. jahrg. 1868. p. $1380 \mathrm{ff}$. p. 626 .

20. Heidelberger jahrbücher der literatur. jahrg. 60. (1867)

21. L. Kayser, Fleck. jahrb. für kl. philol. bd. 97. (1868) p. $805-817$.

22. L. Grasberger zur kritik der römischen archäologie des Dionysios von Halikarnass. Festgruss der philolog. gesellschaft zu Würzburg an die XXVI. versammlung deutscher philologen und schulmänner. Würzburg 1868. 8. p. 9-37.

23. Adolf Kiessling zur kritik der römischen archäologie des Dionysius von Halikarnass. Einladungsschrift zur promotionsfeier des pädagogiums. Basel 1868. 4. p. 20.

24. L. Kayser, Heidelberger jahrbücher der literatur. jahrg. 61. (1868) p. $673 \mathrm{ff}$.

25. Philologus bd. XXIV (1868) p. 374.

26. Adolf Kiessling, Juba und Dionysius von Halikarnass. rhein. museum. n. f. jahrg. XXIII. (1868) p. 672.

27. K. W. Nitzsch quellenanalyse von Livius II, 1-IV, 8 und Dionysius Halicarnassensis $\mathrm{V}, 1-\mathrm{XI}, 63$. rhein. museum. bd. XXIII (1868) p. $600-631$. Erster artikel.

28. K. W. Nitzsch, rhein. museum. bd. XXIV (1869) p. 145-180. Zweiter artikel. 
29. K. W. Nitzsch, rhein. museum. bd. XXV (1870) p. 75-128. Dritter artikel.

30. L. Grasberger, zur kritik des Dionysios von Halikarnass. Philologus bd. XXVIII (1869) p. $344 \mathrm{ff}$. p. $546 \mathrm{ff}$.

31. Dionysi Halicarnasensis antiquitatum romanarum quae supersunt recensuit Adolphus Kiessling. Vol. IV. Lipsiae in aedibus B. G. Teubneri. MDCCCLXX. p. XXXVII. u. 293. 8.

32. Literarisch. Centralblatt f. Deutschland. Herausg. von Fr. Zarncke. jahrg. 1870 . p. $892 \mathrm{ff}$. p. 515 .

33. Heidelberger jahrbücher der literatur. jahrg. 63 (1870)

34. L. Kayser, Fleck. jahrb. für kl. philol, bd. 101 (1870) p. 713-728.

35. H. Usener lectiones graecae, rhein. museum. bd. XXV (1870) p. 610.

36. M. Haupt varia, Hermes bd. 5 (1871) p. 174. 75.

37. Jo. Nic. Madvigii, adversaria critica ad scriptores graecos. Vol. I de arte coniecturali. Emendationes graecae. Hauniae MDCCCLXXI. 8. p. 722-26.

38. Carolus Jacoby observationes criticae in Dionysii Halicarnassensis antiquitates romanas. Acta societ. phil. Lipsiensis ed. Frid. Ritschelius. T. I, p. 287-344. Lipsiae 1872.

39. W. H. Roscher zu Dionysios von Halikarnass. Fleck. jahrb. f. kl. phil. bd. 107 (1873) p. $331 \mathrm{ff}$.

40. C. Peter, Dionys. Hal. ar. III, 68. Philologus bd. XXXIII (1874) p. 572.

41. C. Peter, Dionysius von Halikarnass und Livius. rhein. museum bd. XXIX. (1874) p. $513-560$.

42. Carl Jacoby, über die sprache des Dionysius von Halikarnass in der römischen archäologie. Aarau 1874. p. 38. 4.

43. H. Köstlin, Philologus bd. XXXIV (1875) p. $755 \mathrm{ff}$.

44. J. J. Müller, studien zur römischen verfassungsgeschichte: I. Dionysius II, 7 oder das verhältnis der gentes und curien im alten Rom. Philologus bd. XXXV. (1875) p. 96-136.

45. F. K. Hertlein zu Dionysius Halicarnasensis. Hermes. bd. $\mathrm{X}$ (1876) p. $408-422$.

46. Augustus Tegge quaestionum de Dionysi Halicarnasensis usu praepositionum specimen I. Dissertatio inaugur. philol. Gryphiswaldiae. MDCCCLXXVI. p. 40. 8.

Nachdem wir im ersten theile unseres jahresberichts einen mehr nur flüchtigen blick auf die leistungen für Dionysius bis zum jahre 1860 geworfen haben, wird es sich nun darum bandeln eingehender dasjenige zu prüfen, was seit dem erscheinen des ersten bandes der Kiessling'schen ausgabe und gerade auf grund dieser für die archäologie geschehen ist. Dass hier nicht die rede sein kann von einer eingehendern besprechung dieser ausgabe 
selbst, ist an und für sich klar; schliesst doch eine prüfung der leistungen anderer zugleich eine prüfung dieser ausgabe ein. Mit ausnahme dessen, was wir aus der praefatio des ersten bandes p. V-VII in bezug auf die handschriften schon -oben anführten, enthält dieselbe noch einen sehr kurzen überblick über die leistungen der vorgänger, sowie eine rechtfertigung des standpunktes, den Kiessling in der aufnahme handschriftlicher lesarten eingenommen hat. Es bezieht sich diese rechtfertigende bemerkung namentlich auf orthographische dinge, in denen er nicht alles gleichförmig gestalten konnte und wollte, wie es namentlich Cobet gethan habe. - Ich kann auch hier nur dasjenige wiederholen, was ich schon einmal in bezug auf diese frage (0bservat. crit. p. 290) geäussert habe. Wie damals verwahre ich mich auch hier dagegen alles wie es Cobet und andere thun uniformiren und eine constanti a erstreben zu wollen, die näher betrachtet als eine reine willkür sich darstellt. Ich habe mit absicht die worte des recensenten in den Heidelb. jahrb. 1861 p. 130 gebraucht, der Kiessling seiner inconstantia wegen noch preisen zu müssen glaubt. Es ist selbstverständlich, dass der handschriftlichen autorität bei der herausgabe eines schriftstellers rechnung getragen werden muss, selbstverständlich auch, dass man die natürliche freiheit eines schriftstellers nicht einschränken soll, allein ich habe die feste überzeugung, dass auch ein schriftsteller der spätern zeit wie Dionysius nicht in einem willkürlichen zum theil ungriechischen mischmasch geschrieben haben kann. Im anschluss an Ritschl, Sintenis und Grasberger glaube ich in meinen beiden abhandlungen über Dionysius bereits zur genüge gezeigt zu haben, dass derselbe gewisse ausdrücke und redewendungen beständig gebraucht, dass auch er gewisse gesetze zur vermeidung des hiatus befolgt', bestimmte wortstellungen liebt und dgl. mehr. - Nur zu oft hat die unkenntniss der abschreiber das ursprüngliche verwischt, das wir nur durch sorgfailtige beobachtung des individuellen sprachgebrauchs wiederherstellen können. Uebrigens hat auch Kiessling selbst darüber keine andere ansicht, wie ich obs. crit. p. 290 gezeigt habe, nur dass er nicht immer in seiner ausgabe danach gehandelt hat. Mehreres trägt er deshalb namentlich für die ersten bücher im programm von Basel nach, was in einer zweiten auflage sich gewiss im texte selbst finden wird. Auch darin wird man übrigens Kiessling beistimmen können, wenn er ebendas. p. 10 Cobets verdächtigungen einzelner worte gegenüber sich also äussert: überhaupt sind die stilistischen gesetze des strengen atticismus bei einem schriftsteller wie Dionysius, dessen ganzer stil fast nur aus reminiscenzen bald an Thukydides, bald an Lysias, bald an Demosthenes künstlich zusammengestickt ist, nicht durchzuführen; allein damit soll, denke ich, nicht gesagt sein, dass die schreibweise des Dionysius nicht eine eigenartige ist. In jüngster zeit hat Hertlein im Hermes bd. $\mathbf{X}$ ganz und gar in $\mathbf{C o -}$ 
bets manier den Dionysius behandelt, oder richtiger gesagt, die von Cobet in den Variae und Novae lectiones gewonnenen resultate und gefundenen gesetze auf Dionysius übertragen, zum theil in verkehrter weise, zum theil aber auch, wie wir sehen werden, mit gutem erfolge.

Doch ich kehre zu Kiessling und seiner ausgabe zurück. Die leistungen der vorgänger sind im ganzen fleissig benutzt, ja manchmal im verhältniss zu dem in der adnotatio critica gestatteten raume in einer zu ausgedehnten weise angeführt. Was nützt es ausführlich unmögliche vermuthungen $\mathrm{z}$. b. von Schnelle anzuführen, was schon Sintenis Z. Centralblatt 1865 p. 743 mit recht tadelte. In dieser allerdings etwas scharf gehaltenen beurtheilung des II. bandes aurch Sintenis finden sich aber so viel berechtigte aussetzungen der Kiessling'schen ausgabe, dass es wohl lohnt einige hervorstechende hier anzuführen. Dahin rechne ich den vorwurf der flïchtigkeit, der dort gegen Kiessling erhoben wird, die voreiligkeit unbegründete einfalle sofort in den text aufzunehmen, endlich die ungenauigkeit in der angabe des urhebers und das voranstellen der eignen person. Um gerecht zu sein muss nun andererseits aber auch gleich hier bemerkt werden, dass mehrere änderungen Kiessling im Basl.progr. wie es mir scheint gegen Sintenis glïcklich vertheidigt, andere ausstellungen in den folgenden bänden wenigstens zum theil beherzigt hat. Im gegensatze zu dieser, wie gesagt, scharfen recension hat Kiessling an L. Kayser in den angegebenen bänden von Fleckeisens jahrbüchern einen gar zu milden und gläubigen beurtheiler gefunden, der leicht zu befriedigen war.

Zum schlusse meiner erörterung der Kiessling'schen ausgabe im allgemeinen sei noch bemerkt, dass, wie der vf. p. VII erwähnt, er für den I. band, der buch I-III enthält, durch beiträge von Aug. Meineke, Fr. Bücheler und Usener reich unterstützt wurde; auch Bernays name findet sich an verschiedenen stellen.

Im interesse der übersicht halte ich es nun für den nachfolgenden bericht für geboten, zuerst der zeit nach die erschienenen und oben angeführten leistungen zu besprechen, dann aber um einzelne, namentlich schwierigere stellen, für welche mehrfache vorschläge der heilung vorliegen, nicht öfters behandeln zu müssen, nach buch und capitel die handschriftliche ïberlieferung, und die gemachten vorschläge vorzuführen.

Zu dem, was ich schon oben aus Sauppe's eingehender recension, die allerdings nur das erste buch der archäologie betrifft, in bezug auf die handschriftenfrage angeführt habe, füge ich noch folgendes hinzu: I, $9(11,32)$ und $I, 61(76,8)$ verlangt Sauppe p. 1859 die reflexivformen $\alpha \dot{v} \tau o \tilde{v}$ und $\alpha \dot{v} \tau(\tilde{\varphi}$, wie sie sich bei gleicher ausdrucksweise an andern stellen finden. I, $31(37,31)$ wird noch einmal von Sauppe die unhaltbarkeit des $\mu$ śvo

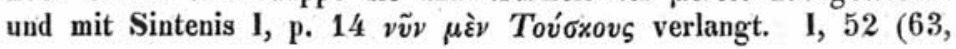




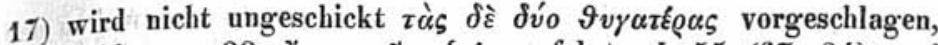

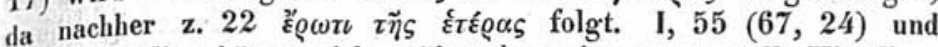
$86(102,16)$ schützen sich, während an der ersten stelle Kiessling, an der zweiten Sintenis l, p. 17 ein doppelt gesetztes عilr verlangt. p. 1863 macht Sauppe auf die verschiedene schreibweise des na-

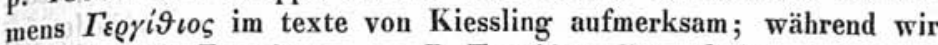
$72(87,31) \Gamma \varepsilon \rho \gamma i \vartheta \iota \varsigma$, wo B $\Gamma \varepsilon \rho \gamma \dot{\eta} \vartheta \iota \varsigma$ hat, finden, steht 49 $(59,1) \Gamma \varepsilon q \gamma \eta \dot{\vartheta} \iota \circ \varsigma$ im texte. Das sind inconsequenzen der schreibweise im texte von Kiessling, die wohl niemand loben kann; ihre

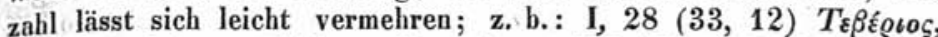
wie cod. A hat. Tıßย́

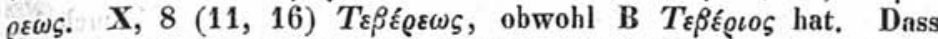
Dionysius ein besonderes wohlgefallen gehabt habe in der schreibweise dieses namens zu wechseln, wird doch wohl niemand glanben, ebenso wenig dass ihm I, $32(38,18) K a \pi \iota \tau \omega \lambda i \omega$, I, $34(40,20)$

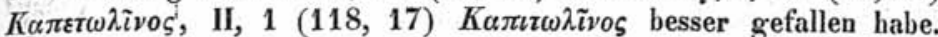

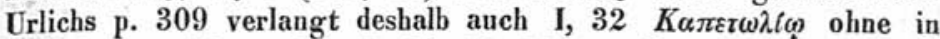

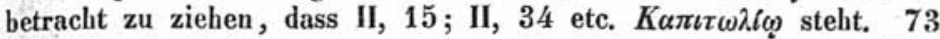

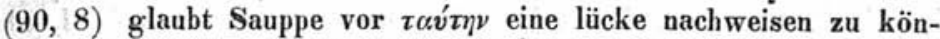

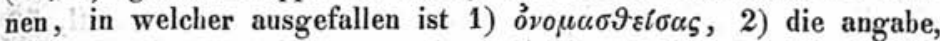
welche städte Romulus gegründet, 3) der anfang einer von der ersten verschiedenen sage, dass schon früher bald nach dem troischen kriege eine stadt Rom gegründet worden sei; mir scheint der nachweis der lücke vollkommen gelungen. $16(19,32)$ sehe ich

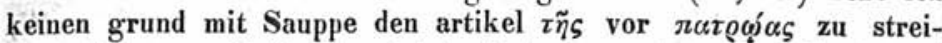
chen; im gegentheil, er scheint mir sogar nothwendig, da ja von ihrem vaterlande die rede ist. Ganz verunglückt ist $20(24,18)$ die erörterung über das digamma. Aus den zwei andern recensionen - die anzeigen in den Heidelberger jahrbüchern enthalten nichts neues und eigenes, so dass sie hier übergangen werden können - bringt eigene vermuthungen Kayser: I, 30 (36, 18) woselbst Kiessling mit Reiske an den ausfall von $\varepsilon x \varepsilon x \tau \eta \eta v o$ dachte,

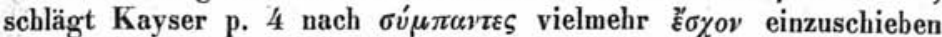
vor. Der ausfall dürfte sich, denke ich, schwer entscheiden lassen.

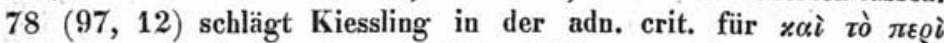

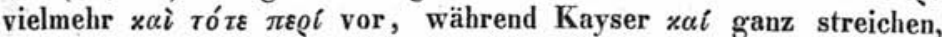
wie auch $59(185,9) \tau \alpha^{\prime}$ tilgen, statt mit Kiessling $\tau \alpha v \tilde{\tau} \alpha$ lesen will. II, $61(187,14)$ soll wieder oi, aus dem Kiessling ' $P \omega$ -

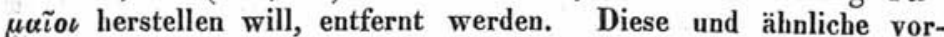
schläge Kayser's in jener recension, die sonst eingehender als alle übrigen ist, entbehren meiner ansicht nach gar zu sehr aller wahrscheinlichkeit, als dass man sie billigen könnte.

Mehr als irgend einer hat C. Sintenis für die wiederherstellung des verderbten textes der archäologie geleistet, so dass mit vollem rechte der mehrzahl seiner vorschläge bereits eine stelle im texte bei Kiessling angewiesen ist. In ähnlicher weise, wie in seinem 
ersten programme bemüht sich Sintenis auch in diesem zweiten det beweis zu liefern, dass, um dem Dionysius zu seinem eigenthume zu verhelfen, beide handschriften zu rathe gezogen, die lesarten sorgsam geprüft und die wahrscheinlicheren aufgenommen werden müssen. Nur so, indem man zugleich sorgfältig die fehler des cod. Urbinas beachtet, aus denen sich oftmals mit geringer änderung die wahre lesart gewinnen lässt, könne ein gereinigter text gewonnen werden. Diese beiden behauptungen werden nun durch eine reihe von ähnlichen fällen, die zusammengestellt werden, gestützt und erwiesen, den fehlern aber nach möglichkeit abgeholfen. Da diese abhandlung nach dem erscheinen von band I. der Kiessling'schen ausgabe geschrieben ist, so sind die das I-III. buch berührenden vorschläge bis jetzt noch nicht zur verwerthung gelangt. Ich beginne also mit den diesen büchern entnommenen stellen, die mir unbedingt späterhin aufnahme zu verdienen scheinen: I, $56(69$,

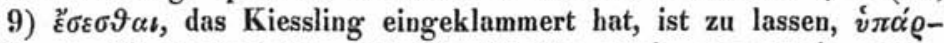

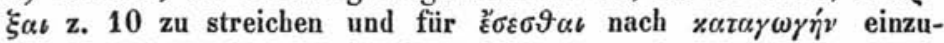
setzen; die verderbniss ist durch die vertauschung der synonyma

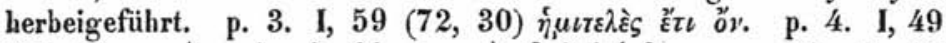

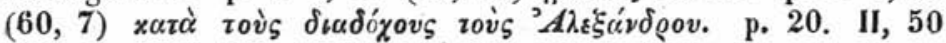

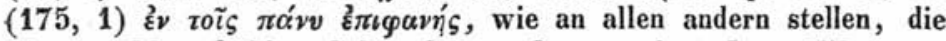
Sint. p. 32 aufzählt. Schon Struve Opusc. select. I. p. 173 nennt

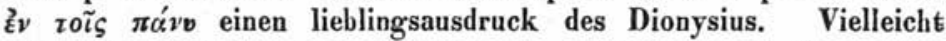
wird man auch die verbesserungen zu II, 14 und II, 42 in den text aufnehmen können. Aus dem dritten buche erwähne ich: III, $6(217,28)$ v $\pi 0 \vartheta \varepsilon \varepsilon \varepsilon \varepsilon$ in $v_{\pi j o ̀} \vartheta \varepsilon i \alpha \varsigma$ geändert (p. 4) das jedoch Sintenis durch Cobet Mnemosyne IX vorweggenommen ist. III, 40 $(277,27) \alpha v^{2} \iota \varsigma$, das sich auf III, 33 zuriuck bezieht p. 11.

In welch' ausgedehnter weise Kiessling in den nächsten drei bänden von den vorschlägen von Sintenis gebrauch machen konnte und auch gemacht hat, beweist zur genüge folgende aufzählung:

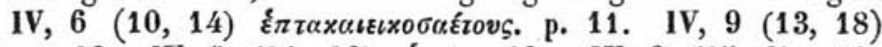
ovjoc. p. 12. IV, $9(14,10)$ ws. p. 12. IV, $9(15,8)$ steht

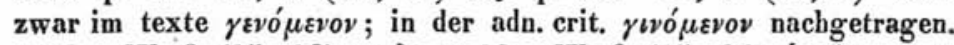

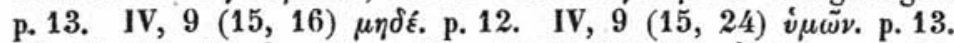

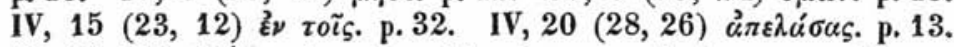
IV, $26(38,25)$ xøeizzovi. p. 14, schon von Portus vorgeschlagen. IV, $29(43,16)$ wird $\pi \varepsilon \rho l \varepsilon \sigma \tau \iota v$ in der adn. crit. nachgetragen.

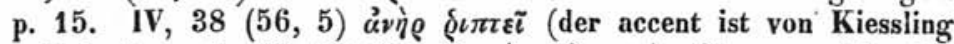

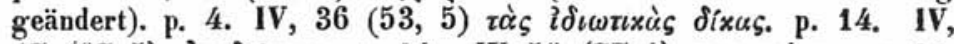

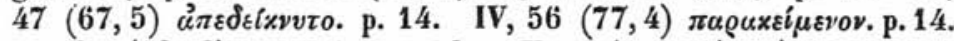

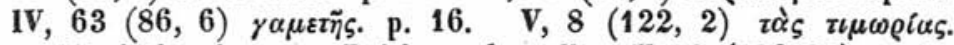
p. 17, doch schon von Reiske aufgestellt. V, $13(129,21)$ ตv $\tilde{\eta} \varsigma$. p. 18, worin er ebenfalls Reiske folgte. V, $23(140,18) \sigma \tau u ́-$

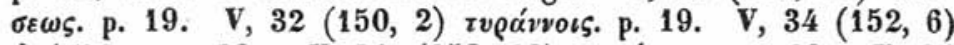

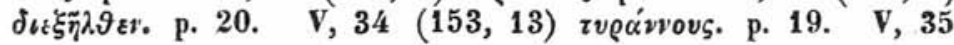




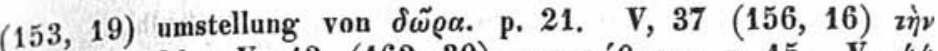

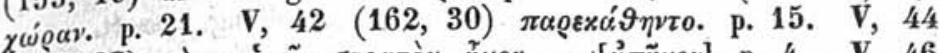

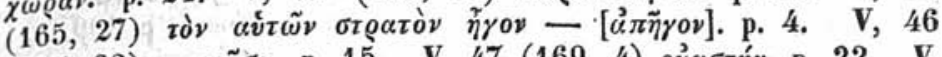

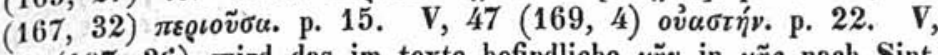
$60(187,26)$ wird das im texte befindliche $\gamma \tilde{\eta} \nu$ in $\gamma \tilde{\eta} s$ nach Sint. p. 11 corrigirt in dem druckfehlerverzeichniss (vgl. dagegen meine Observat. crit. p. 319). V, $65(192,28)$ ż́ $\varepsilon^{2}$ p.23. V, 65 (193,

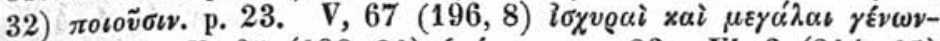

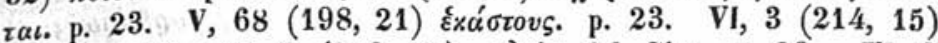
ixuví. p. 25. VI, $5(216,12) \alpha v^{2} \eta^{\prime} v$ del. Sint. p. $26 . \quad$ VI, 9

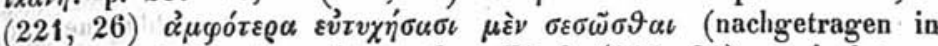
der adn. crit.) $x \alpha i$ vixãv. p. 9 . VI, 9 (221, 31) $\pi \alpha \varrho$ ' $\varepsilon \varepsilon \varrho \omega v$. p. 9, sowie die annahme der lücke nach $\alpha \xi \xi \omega \varsigma$. p. 9 . VI, 9 (222,

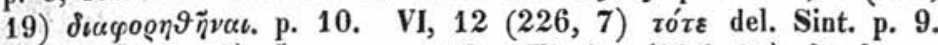

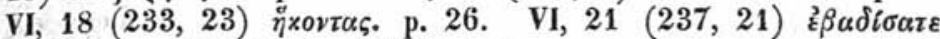

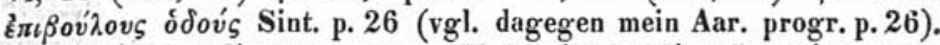

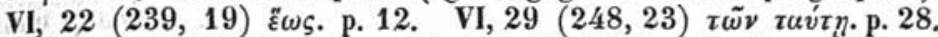

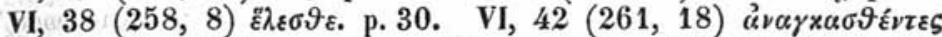

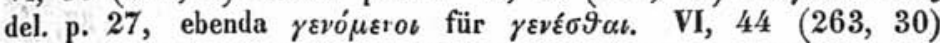

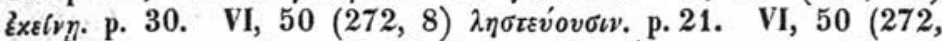
12) $\beta \varepsilon \beta \alpha i \omega c$. p. 22 . VI, $50(272,13) \pi v \sigma \tau \iota v . ~ p . ~ 22 . \quad$ VI, 50 $(272,21) x \alpha i$ eingeklammert von Sint. p. 22 . VI, $53(275,29)$

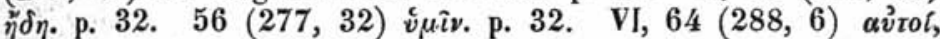
wie auch cod. $C$ hat, p. 30 . VI, $64(288,22)$ ti eingeklammert

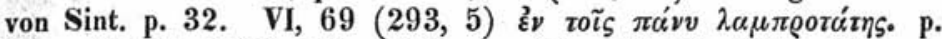
33. VI, 71 (295, 12) $x \omega \lambda$ ṽov. p. 33. VI, $75(301,31) \tau \alpha_{\varsigma}$

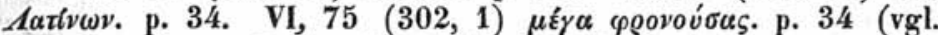

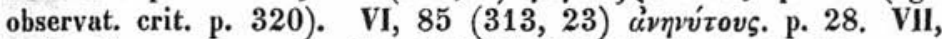

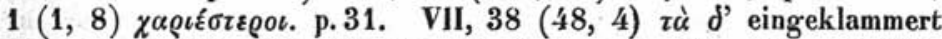
von Sint. p. 33. VII, $41(50,1)$ ä $\pi \alpha v \tau \varepsilon$. . p. 36. VII, 41 (50,

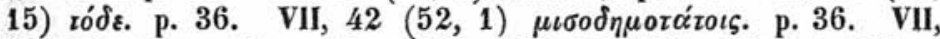

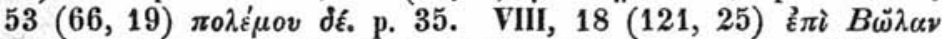

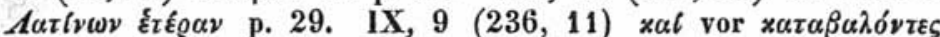
rührt von Sint. p. 35 her. IX, $45(291,20) \pi \alpha \varrho \iota v \tilde{\sigma} \sigma \alpha$. p. 4. IX,

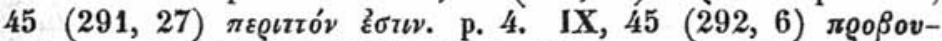

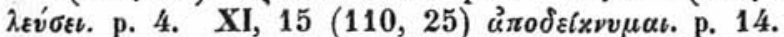

An diesen stellen also und einigen andern ist Kiessling den vorschlägen von Sintenis gefolgt und zwar zum grössten theile wohl mit recht. Einige, über welche ich eine andere ansicht habe, werde ich nachher behandeln. Die grosse belesenheit im Dionysius und die kenntniss seines eigenthümlichen sprachgebrauchs ermöglichten Sintenis, zumal auch ihm die collationen der handschriften durch Ritschl zur verfügung standen, den meisten seiner vorgeschlagenen änderungen durch eine reiche zahl von beispielen gewicht zu verleihen. Zum schlusse meiner besprechung erwähne 
ich noch, dass auch der referent in Zarncke's centralblatt 1862 p. 557 sich nur anerkennend über diese abhandlung äussert.

Die wenigen bemerkungen, welche Urlichs Eos I, p. 309. 324 zu dem ersten buche des Dionysius macht, sind wie wir aus einer anmerkung erfahren, aus dem philologischen seminar hervorgegangen. Auffallend genug ist es dabei nur, dass auf frühere änderungen anderer nicht die mindeste rücksicht genommen, ja nicht einmal die adnotatio critica von Kiessling eingesehen wird. 1, 4 (6,

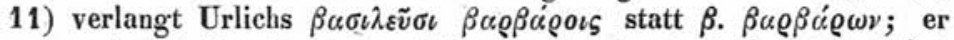
weiss also nicht, dass der dativ in den handschriften überliefert ist; im übrigen hat er recht, die von Bernays vorgeschlagene und von Kiessling aufgenommene änderung $\beta \alpha \rho \beta \alpha \dot{\alpha} \rho \nu$ ist zu verwerfen.

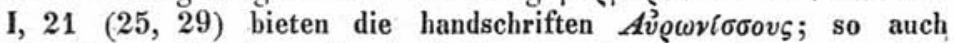
Kiessling. Urlichs verlangt Av̉@oú yxovs, ohne zu wissen oder hinzuzusetzen, dass Sylburg und Portus schon so lesen wollten; auf das nun gar, was W. Fröhner Philologus XII (1856) p. 217 in

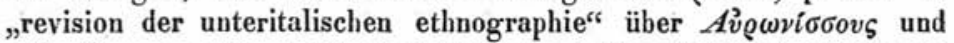
Av̉œov́yxovৎ sagt, wird natürlich gar keine rücksicht genommen. Die einzige änderung, der man beistimmen kann, ist I, $31(37,4)$

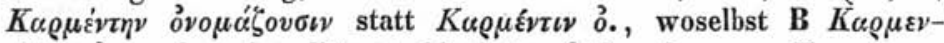

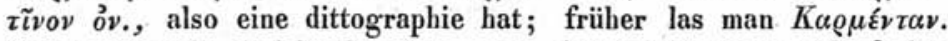

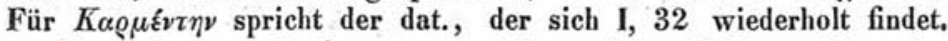
- Nach einem verlaufe von vier jahren d.h. 1864 erschien der zweite band der Kiessling'schen ausgabe, der wiederum drei bücher IV, V, VI umfasst und in derselben weise gearbeitet ist, wie der erste. In bezug auf die lesarten des Urbinas beansprucht Kiessling von nun an grössere glaubwürdigkeit, als Hudson's ausgabe, die man ihm wohl, da er den cod. B selbst von neuem in Rom verglichen hat, wird einräumen müssen; auch unterscheidet er nun zwischen $\mathbf{B} a$ und $\mathbf{B} b$, womit er die zwei verschiedenen hände in cod. B bezeichnet. Aus der ausfübrlichen besprechung, die dieser band durch L. Kayser, der in die von Sintenis, Zarncke's centralblatt 1865 p. 742 , erhobenen vorwürfe nicht einstimmen kann, erfahren hat, wäre folgendes anzuführen: VI, $29(248,17)$ hat

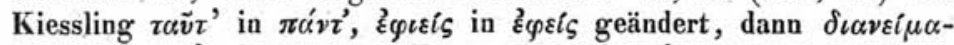

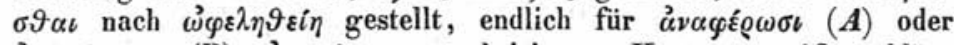

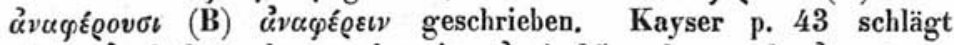
statt $\omega^{3} \varphi \varepsilon \lambda \eta \vartheta \varepsilon i \eta$ den conjunctiv $\omega^{3} \varphi \varepsilon \lambda \eta \vartheta \tilde{\eta}$, also auch $\dot{\omega} \nu \alpha \varphi \xi \rho \omega \sigma \iota$

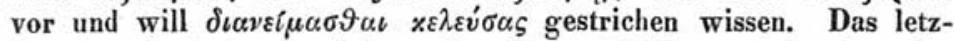
tere ist ein kurzes verfahren, das niemand billigen wird; ohne eine heilung der periode zu wissen, scheint mir so viel sicher, dass

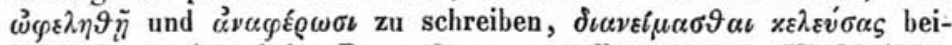
zubehalten sein wird. Den schwer entstellten worten VI, 32 (251, $27)$; VI, $34(253,22)$; VI, $36(256,6)$ ist durch Kayser p. 43. 44 nicht im mindesten hülfe gebracht. Auch andere vorschläge, die Kayser daselbst vorbringt, um verderbte stellen des VII. buches zu 
heilen, befriedigen mich wenigstens nicht. VI, $56(278,32)$ er-

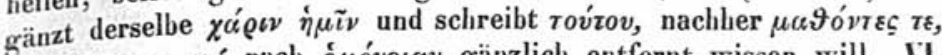
wogegen er $x a i$ nach ó $\mu o ́ v o \iota \alpha \nu$ gänzlich entfernt wissen will. VI, 61 (284, 20) soll mit A B C $\pi о \iota \sigma^{\prime} \varepsilon \tau \varepsilon$ beibehalten, z. $22 \pi \varepsilon \rho \iota \sigma \eta^{\prime}-$

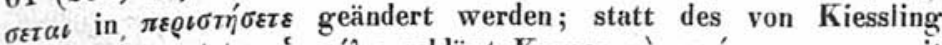

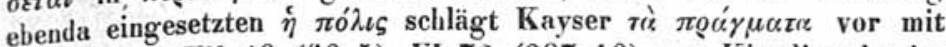
hinweis auf VII, $49(60,5)$. VI, $72(297,10)$, wo Kiessling in der

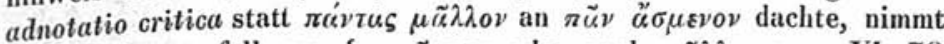
Kayser den ausfall von $\varepsilon \alpha v \tau o \tilde{v}$ vor oder nach $\mu \tilde{u} \lambda \lambda o v$ an. VI, 79

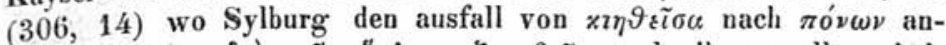

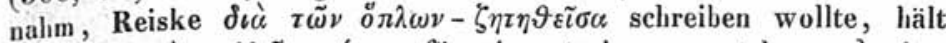

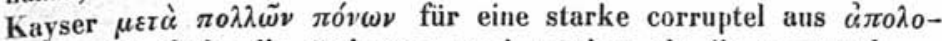
$\mu \xi \mathfrak{\eta}$; ich finde die änderung noch stärker als die corruptel. Für das IV. und V. buch, die weniger als VI. gelitten haben, bringt Kayser ebenfalls einige wenige vorschläge : IV, $2(2,16)$ schiebt

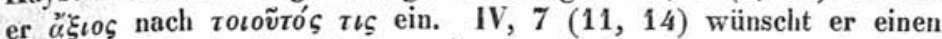

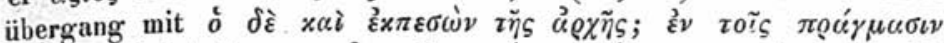

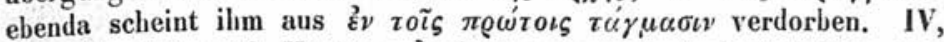

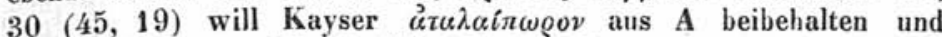

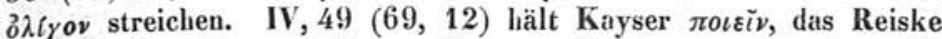

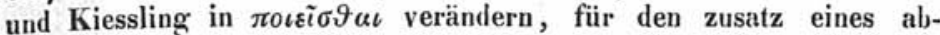

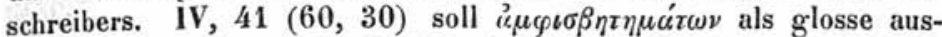

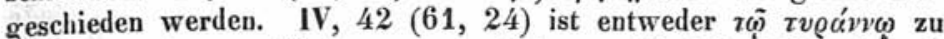
streichen oder $\chi \alpha \varrho \zeta \zeta \mu \varepsilon v o s$ einzuschieben. VI, $75(301,4)$ soll ebenfalls einen zusatz von ė $\pi \iota \delta \iota x \nu v ́ \mu \varepsilon v o \iota$ oder einem ähnlichen

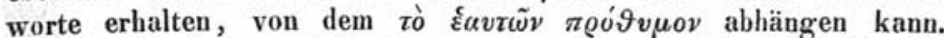

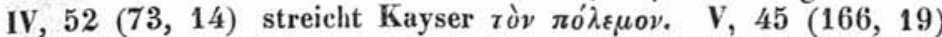

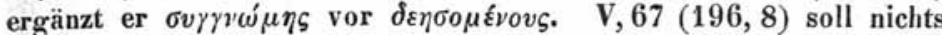

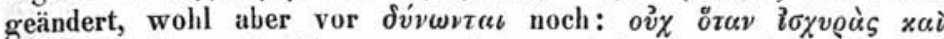

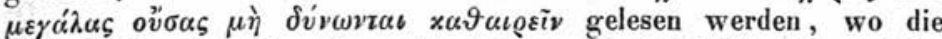

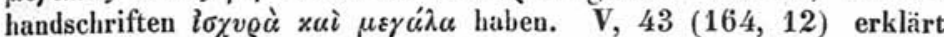

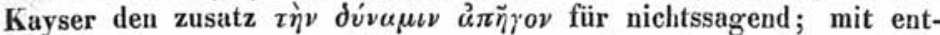

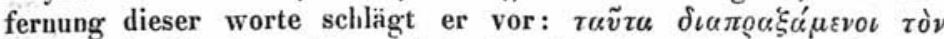

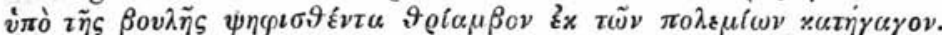

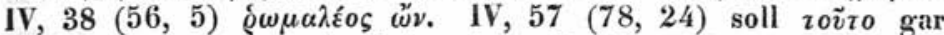

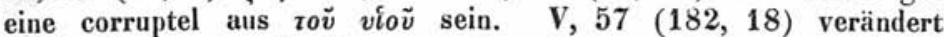

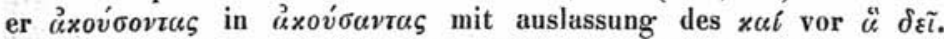

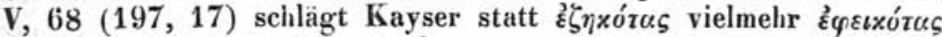

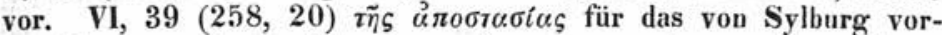

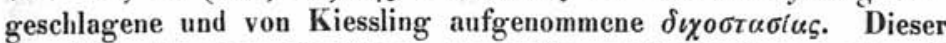
vorschlag ist schon deshalb zu verwerfen, weil wie Sintenis III, p. 2

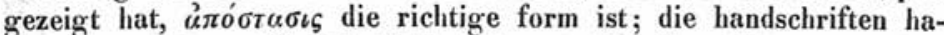

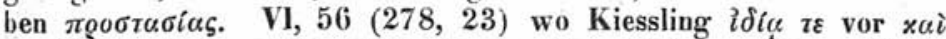
xoเvñ einschieben will, hält Kayser für richtiger $x a i$ xoıv $\tilde{\eta}$ als

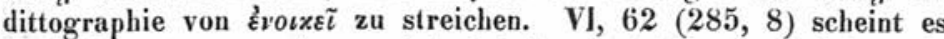
ihm leichter $\tau \varepsilon x \mu \alpha \iota \rho o ́ \mu \varepsilon \nu o \varsigma$ beizubehalten und ó zu streichen als

Philologus. XXXVI. bd. 1. 


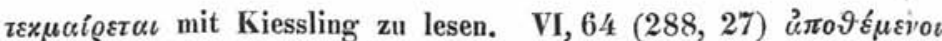

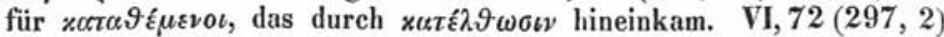

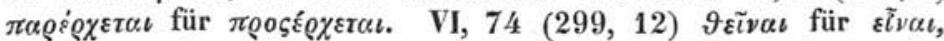

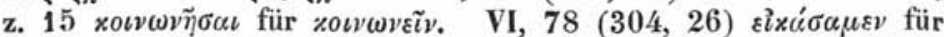

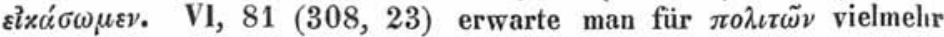

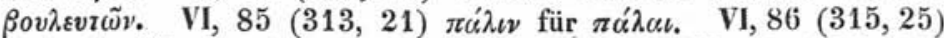

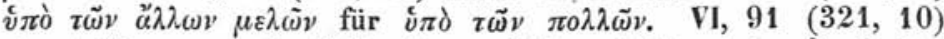
nimmt Kayser an $\dot{c}^{3} \pi \circ \delta \varepsilon i \xi \alpha \sigma \vartheta \alpha \iota$, auf das gleich wieder $\stackrel{3}{\alpha} \pi 0 \delta \varepsilon i \xi \alpha-$

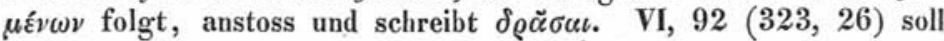

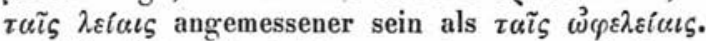

Im III. und letzten specimen emendationum Dionysiacarum, von dem mir keine recension bekannt geworden ist, behandelt C. Sintenis vorzugsweise stellen aus den büchern VII - XI, und nur wo:-ce bemerkungen berülıren die sechs vorangehenden. I, 82 (104, 25) fügt er am schlusse des capitels $\delta \iota \alpha \tau \varepsilon \lambda \varepsilon \tilde{\imath}$ hinzu. p. 7. I, 40

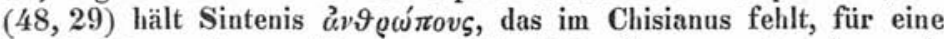

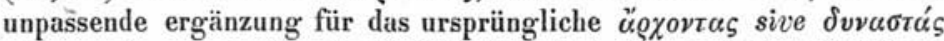

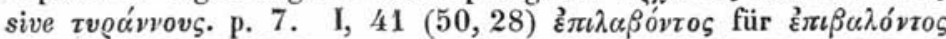
mit anführung ähnlicher stellen. p. 11. II, $71(199,22)$ wird mit sehr leichter änderung dem Dionysius sein eigenthum dadurch wie-

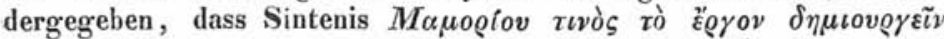

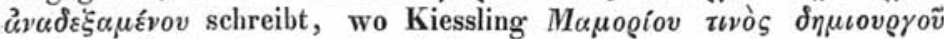
zò éoyov $\vec{a}$. in den text aufnahm, wie Portus die worte umgestellt und verändert hatte. Eine angabe in der adn. critica fehlt p.4.

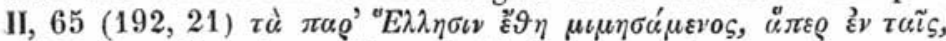
p. 22, woselbst ferner über die häufige verwechselung von $\pi \alpha \varrho \alpha$ und $\pi \varepsilon \varrho$ gehandelt wird. II, $45(169,1) \pi \alpha \varrho \tilde{\eta} \lambda \vartheta o v$ für $\pi \varrho o \tilde{\eta} \lambda \vartheta o v$. p. 22. Die verschreibung von $\pi \mu \rho \alpha-\pi \rho o-\pi \rho \circ$ wird an beispielen nachgewiesen und verbessert. III, 37 (274, 15) $\pi 0 \lambda \varepsilon-$

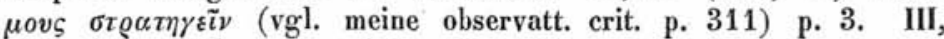
$49(287,15) \dot{a} \pi \tilde{\eta} \gamma \varepsilon-\eta_{\gamma \varepsilon}$ hat schon Kiessling in der adn. crit.

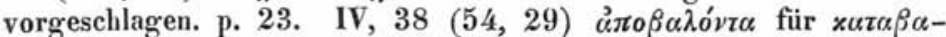

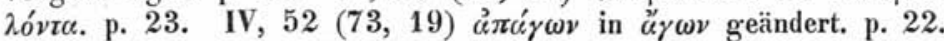
V, $39(159,5)$ hat Kiessling mit Reiske $\tau o ́ \pi \omega$ eingeklammert,

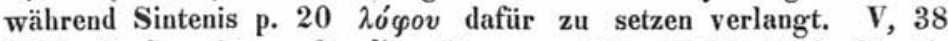

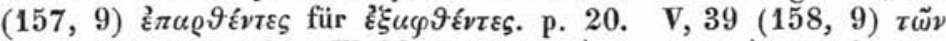

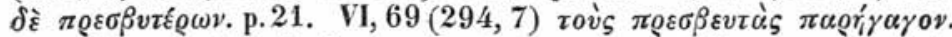

Die vorzüglichkeit der verbesserungsvorschläge von Sintenis für die folgenden bücher glanbe ich wiederum nicht besser darthun zu können, als dadurch, dass ich die von Kiessling in den text aufgenommenen hier in gleicher weise wie oben aufführe: VII, (1,

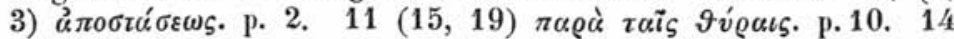

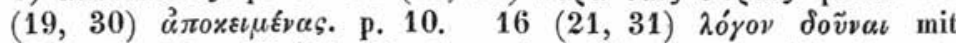

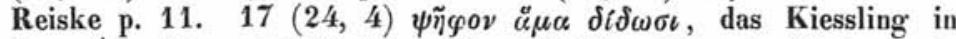
der adn. critica nachträgt. p. $13.19(26,19)$ schreibt im texte

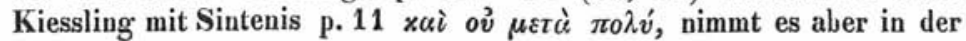


adn. critica zurück. Vgl. mein Aar. progr. p. $19.29(37,7)$

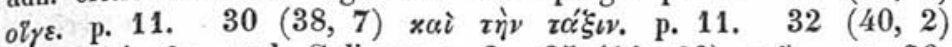

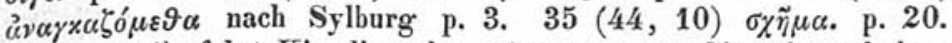
$33(52,14)$ folgt Kiessling der erörterung von Sintenis und deutet die lücken an. p. $9.47(58,2) \pi u \varrho \iota \sim \tilde{v} \sigma \iota$. p. $12.50(61,2)$

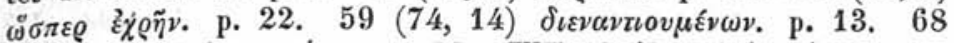

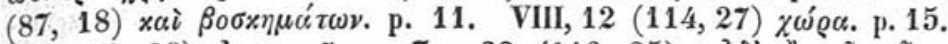

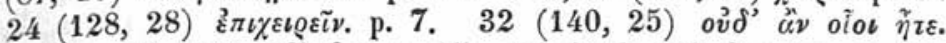

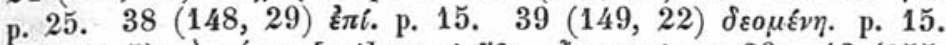

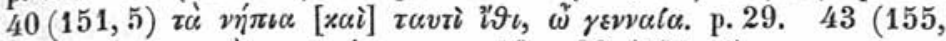

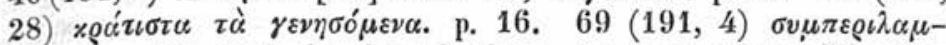

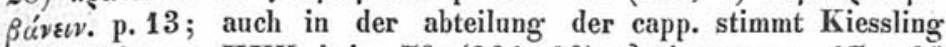
adn. critica p. XXII bei. $79(204,10)$ ảmó $\varphi \alpha \sigma \iota v$. p. 17. 86

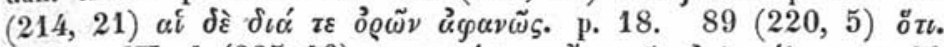

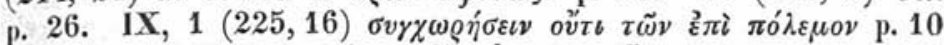

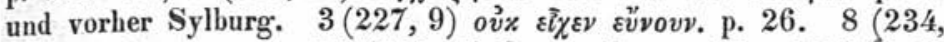

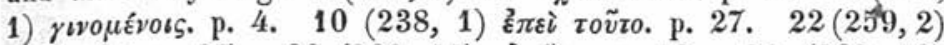

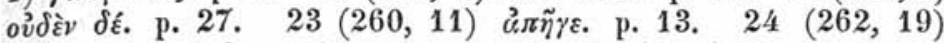

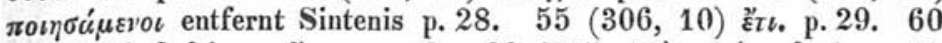

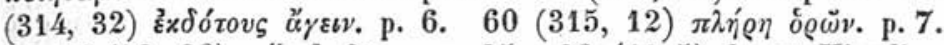

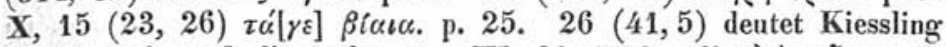
mit Sintenis p. 9 die lücke an. XI, $22(120,13)$ àvì $\eta^{2} v$. p. 6.

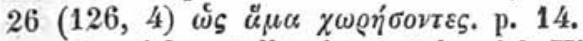

An vielen stellen beschränkt sich Kiessling darauf nur in der adnotatio critica der vorgeschlagenen änderung erwähnung zu thun, ohne dieselbe sofort in den text aufzunehmen, vielfach mit recht. Ich verzichte darauf auch diese stellen hier anzuführen und mache nur noch auf einige lehrreiche erörterungen aufmerksam: p. 3 bespricht Sintenis die häufige verwechselung von $\omega \nu$ und $\varepsilon \iota \nu$, ó $\mu \varepsilon v o \iota$ und $o^{\prime} \mu \varepsilon 9 \alpha$, p. 4. $\eta \varsigma$ - oıs. Ueber vertauschung von synonymen worten handelt er p. 5 ; über lücken p. 5 ff.; über fehler, die durch abirren der augen etc. entstanden sind p. 18; über die fehlerhafte wiederholung von präpositionen p. 22.

Das letzte, was von Sintenis über Dionysius veröffentlicht ist, ist im Hermes I, p. 471 erschienen. An der ersten stelle verbes-

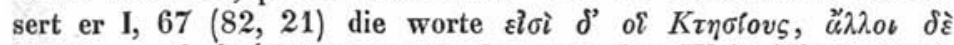
Mvxiovs, of $\delta \check{~ ' E \varrho x \varepsilon i o v \varsigma ~ n a c h ~ d e n ~ v o n ~ J o s . ~ K l e i n ~(F l e c k . ~ j a h r b . ~}$ 89 p. 338) aus den schol. Bernens. (Verg. Georg. I, 498) angeführten worten: indigetes a Latinis qui a Graecis ennichi dicuntur

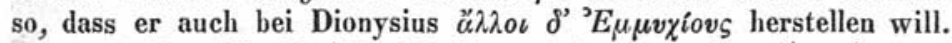

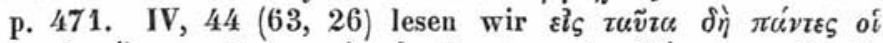

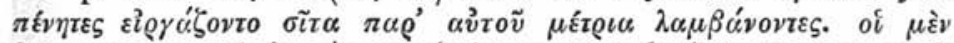

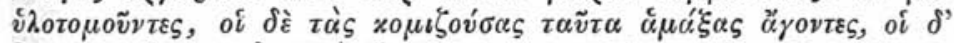

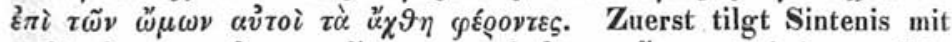
recht $\varepsilon \hat{l} \zeta$ am anfange; die worte of $\delta \bar{\varepsilon}-\alpha^{\prime} \gamma o v \tau \varepsilon \varsigma$ sind aus Urb. und Chis. hinzugekommen, wäbrend in den andern handschriften, 
wie so oft bei mehrgliedrigen eintheilungen gleichen umfangs ein glied von den abschreibern übersprungen worden ist. Im weitern zeigt Sintenis, dass der ganze gedanke zu eng und einseitig ist; v่ unentbehrliches material steine, wie auch nachher neben den $\tau \dot{\varepsilon} \times \tau_{0-}$

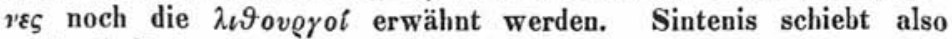

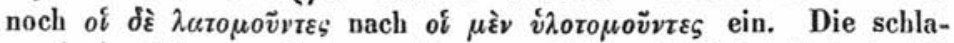
gende bestätigung dieser ergänzung sieht und findet Sintenis IV, $81(105,15)$, wo es mit bezug auf das hier erzählte heisst:

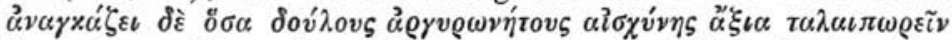

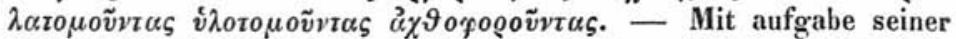
eigenen (spec. II, p. 7) ausgesprochenen vermuthung will Sintenis

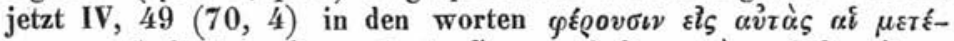

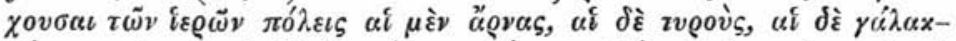

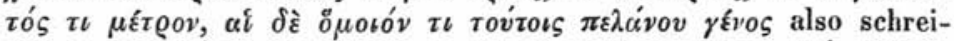

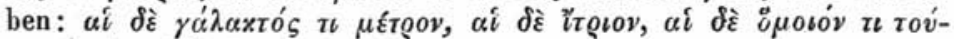

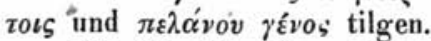

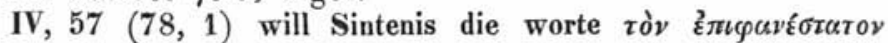
$\tau \tilde{\omega} \nu \Gamma \alpha \beta i \omega \nu$ aus dem texte entfernen und ebenda z. 23 hält er den

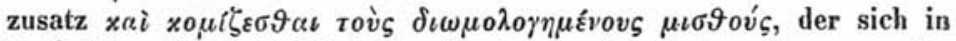
B findet, für bedenklich, weil ein unerträglicher pleonasmus im gedanken entstehe. Endlich streicht er das von Kiessling ex ingenio

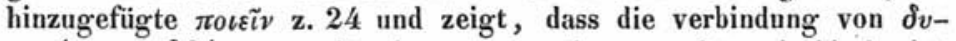

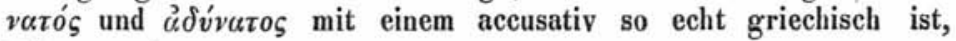
wie nur eine.

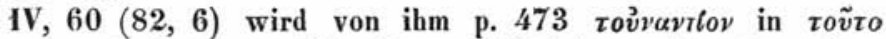

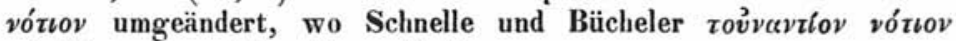
schrieben, was Kiessling aufnahm.

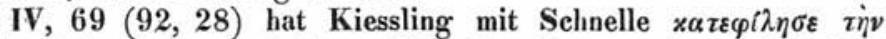
$\gamma \tilde{\eta} \nu$ in $\dot{\varepsilon} \varphi(\lambda \eta \eta \sigma \varepsilon$. $\gamma$. geändert. Sintenis will $x \alpha \tau \varepsilon \varphi i \lambda \eta \eta \varepsilon \varepsilon$ beibehalten, da es sich ebenfalls in den fragmenten des Dio Cassius (A. Mai nova collect. II, 140) findet, wo es von derselben sache lautet:

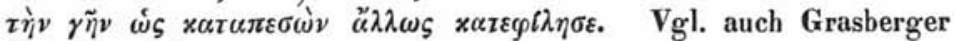
festgr. p. 21.

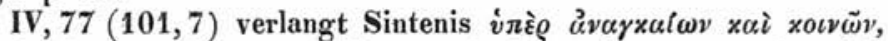
was durch die schlussworte dieses und die anfangsworte des folgenden capitels bewiesen wird.

(Schluss folgt.)

\section{Zu Plutarch.}

Plut. Mor. (de Fort. c. 2) p. 223 Teubn.: $\grave{\eta} \gamma \alpha \grave{g} \sigma \omega \varphi \varrho o \sigma u ́ v \eta$

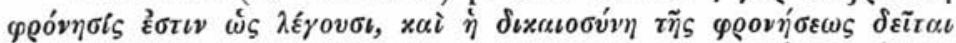

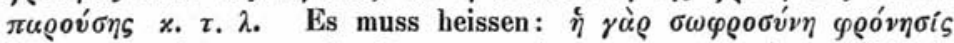

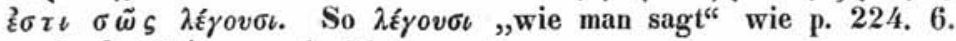

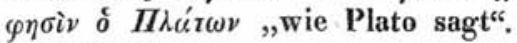

Hamburg.

Heinrich Köstlin. 